QUARTERLY OF APPLIED MATHEMATICS

VOLUME LXV, NUMBER 2

JUNE 2007, PAGES 281-315

$\mathrm{S} 0033-569 \mathrm{X}(07) 01053-8$

Article electronically published on April 25, 2007

\title{
ON THE CAUCHY PROBLEM OF THE BOLTZMANN AND LANDAU EQUATIONS WITH SOFT POTENTIALS
}

\author{
BY
}

LING HSIAO (Academy of Mathematics and Systems Science, Chinese Academy of Sciences, Beijing 100080, People's Republic of China)

AND

HONGJUN YU (School of Mathematical Sciences, South China University of Technology, Guangzhou 510640, People's Republic of China)

Abstract. Global classical solutions near Maxwellians are constructed for the Boltzmann and Landau equations with soft potentials in the whole space. The construction of global solutions is based on refined energy analysis. Our results generalize the classical results in Ukai and Asano (Publ. Res. Inst. Math. Sci. 18 (1982), 477-519) to the very soft potentials for the Boltzmann equation and also extend the classical results in Caflisch (Comm. Math. Phys. 74 (1980), 97-107), Guo (Comm. Math. Phys. 231 (2002), 391-434), and Guo (Arch. Rat. Mech. Anal. 169 (2003), 305-353) in the periodic box to the whole space for the Boltzmann equation and the Landau equation in the Coulomb interaction.

1. Introduction. We consider the Cauchy problem of the Boltzmann and Landau equations with soft potentials for dynamics of dilute particles. The equation considered in this paper reads as

$$
\partial_{t} F+v \cdot \nabla_{x} F=Q(F, F),
$$

where $F(t, x, v)$ is the distribution function of the particles at time $t \geq 0$, located at $x=\left(x_{1}, x_{2}, x_{3}\right) \in \mathbf{R}^{3}$ with velocity $v=\left(v_{1}, v_{2}, v_{3}\right) \in \mathbf{R}^{3}$. The collision between particles is given by the standard Boltzmann collision operator,

$$
Q(F, G)(v)=\int_{\mathbf{R}^{3}} \int_{\mathbf{S}^{2}}\left[F\left(u^{\prime}\right) G\left(v^{\prime}\right)-F(u) G(v)\right] B(|u-v|, \vartheta) d u d \omega,
$$

where $F(u)=F(t, x, u)$ etc., $\omega \in \mathbf{S}^{2}$, the unit sphere in $\mathbf{R}^{3}$, and

$$
v^{\prime}=v-[(v-u) \cdot \omega] \omega, \quad u^{\prime}=u+[(v-u) \cdot \omega] \omega
$$

Received January 15, 2006.

2000 Mathematics Subject Classification. Primary 35Q99; Secondary 35A05.

Key words and phrases. Boltzmann equation, Landau equation, soft potentials, global solutions.

E-mail address: hsiaol@amss.ac.cn

E-mail address: yuhj2002@sina.com

(C)2007 Brown University

Reverts to public domain 28 years from publication 
denote the velocities of particles after the collision, with $u$ and $v$ being their velocities before the collision.

For the interaction potentials of inverse power laws, the collision kernel $B(|u-v|, \vartheta)$ takes the form $5,10,26$

$$
B(|u-v|, \vartheta)=B(\vartheta)|u-v|^{\gamma}, \quad \cos \vartheta=((u-v) \cdot \omega) /|u-v|, \quad-3<\gamma \leq 1
$$

where it is customary to assume that $B(\vartheta)$ satisfies the Grad angular cutoff assumption, $0<B(\vartheta) \leq$ const. $|\cos \vartheta|$. The exponent $\gamma$ is related to the models of potentials of intermolecular forces, namely, the soft potentials $(-3<\gamma<0)$, Maxwellian molecules $(\gamma=0)$, the hard potentials $(0<\gamma<1)$ and the hard sphere model $(\gamma=1, B(\vartheta)=$ const. $\cos \vartheta)$. We are concerned with the soft potentials $-3 \leq \gamma<0$.

For the Coulomb interaction, the Boltzmann equation becomes inadequate because grazing collisions become preponderant over all other collisions. In 1936, Landau derived from the Boltzmann equation another equation in which only grazing collisions are taken into account written as (1.1), but the collision operator is given by

$$
\begin{aligned}
Q(F, G)(v) & =\nabla_{v} \cdot\left\{\int_{\mathbf{R}^{3}} \phi\left(v-v^{\prime}\right)\left[F\left(v^{\prime}\right) \nabla_{v} G(v)-F(v) \nabla_{v^{\prime}} G\left(v^{\prime}\right)\right] d v^{\prime}\right\} \\
& =\sum_{i, j=1}^{3} \partial_{i} \int_{\mathbf{R}^{3}} \phi^{i j}\left(v-v^{\prime}\right)\left[F\left(v^{\prime}\right) \partial_{j} G(v)-F(v) \partial_{j} G\left(v^{\prime}\right)\right] d v^{\prime}
\end{aligned}
$$

where $\phi(w)$ is the positive-semi-definite matrix that takes the general form

$$
\phi(w)=B(|w|) S(w),
$$

where $B$ is a function depending on the nature of the interaction between the particles, and $S(w)$ is the $3 \times 3$ matrix

$$
S(w)=I_{3}-\frac{w \otimes w}{|w|^{2}},
$$

which is the orthogonal projection onto the orthogonal plane to $w$ and $I_{3}$ is the identity matrix of order 3 . When an interaction force $\Re$ between the particles depends on the inter-particle distance $r$ according to an inverse power law $\Re=r^{-s}$ with $s \geq 2$, the function $B$ takes the form $B(|w|)=|w|^{\gamma+2}$ where $\gamma=\frac{s-5}{s-1}$. This leads to the usual classification in terms of hard potentials $(\gamma>0)$, Maxwellian molecules $(\gamma=0)$ or soft potentials $(\gamma<0)([6,18,29])$. The original Landau collision operator for the Coulomb interaction corresponds to the case $\gamma=-3$. The present study with the Landau equation is restricted to the very soft potentials $-3 \leq \gamma<-2$.

We denote a normalized global Maxwellian by $\mu(v) \equiv e^{-|v|^{2}}$. We define the standard perturbation $f(t, x, v)$ to $\mu(v)$ as $F=\mu+\sqrt{\mu} f$. We plug this perturbation into (1.1) to derive a perturbation equation for $f(t, x, v)$. Equation (1.1) for the perturbation $f(t, x, v)$ takes the form

$$
\partial_{t} f+v \cdot \nabla_{x} f+L f=\Gamma(f, f),
$$

with $f(0, x, v)=f_{0}(x, v)$. $L$ is the linear part and $\Gamma(f, f)$ is the nonlinear part. 
For the Boltzmann equation, the linearized collision operator $L f=\nu(v) f-K f$ and notice that $K=K_{2}-K_{1}$ is given by [5, 9 :

$$
\begin{gathered}
\nu(v)=\int_{\mathbf{R}^{3} \times \mathbf{S}^{2}}|v-u|^{\gamma} \mu(u) B(\vartheta) d u d \omega, \\
{\left[K_{1} g\right](v)=\int_{\mathbf{R}^{3} \times \mathbf{S}^{2}}|u-v|^{\gamma} \mu^{1 / 2}(u) \mu^{1 / 2}(v) g(u) B(\vartheta) d u d \omega,} \\
{\left[K_{2} g\right](v)=\int_{\mathbf{R}^{3} \times \mathbf{S}^{2}}|u-v|^{\gamma} \mu^{1 / 2}(u)\left[\mu^{1 / 2}\left(u^{\prime}\right) g\left(v^{\prime}\right)+\mu^{1 / 2}\left(v^{\prime}\right) g\left(u^{\prime}\right)\right] B(\vartheta) d u d \omega .}
\end{gathered}
$$

Notice that for the soft potentials, $\nu(v)$ behaves like $(1+|v|)^{\gamma}$. The nonlinear collision operator $\Gamma(f, g)=\frac{1}{\sqrt{\mu}} Q(\sqrt{\mu} f, \sqrt{\mu} g)$.

For the Landau equation, the linearized collision operator $L$ is defined by $L f=-A f-$ $K f$, where

$$
A f=\frac{1}{\sqrt{\mu}} Q(\sqrt{\mu} f, \mu), \quad K f=\frac{1}{\sqrt{\mu}} Q(\mu, \sqrt{\mu} f)
$$

and the bilinear collision operator $\Gamma(f, g)$ is given by $\Gamma(f, g)=\frac{1}{\sqrt{\mu}} Q(\sqrt{\mu} f, \sqrt{\mu} g)$.

As expected from the H-theorem, $L$ is nonnegative and for every fixed $(t, x)$ the null space of $L$ is given by the five-dimensional space $(1 \leq j \leq 3)$

$$
N=\operatorname{span}\left\{1, v_{j} \sqrt{\mu},|v|^{2} \sqrt{\mu}\right\} .
$$

We define the orthogonal projection from $L^{2}\left(\mathbf{R}^{3}\right)$ onto the null space $N$ by $\mathbf{P}$.

Notation. For notational simplicity, we shall use $\langle\cdot, \cdot\rangle$ to denote the standard $L^{2}$ inner product in $\mathbf{R}^{3}$ and $(\cdot, \cdot)$ to denote the standard $L^{2}$ inner product in $\mathbf{R}^{3} \times \mathbf{R}^{3}$. For the Boltzmann equation, we introduce a weight function of $v$ as $w_{1}(v)=[1+|v|]^{\gamma}$, while we introduce another weight function of $v$ as $w_{2}(v)=[1+|v|]^{\gamma+2}$ for the Landau equation. We always denote the two weight functions as $w=w(v)$.

We denote the weighted $L^{2}$ norm as

$$
|g|_{2, \theta}^{2} \equiv \int_{\mathbf{R}^{3}} w^{2 \theta} g^{2} d v, \quad\|g\|_{2, \theta}^{2} \equiv \int_{\mathbf{R}^{3} \times \mathbf{R}^{3}} w^{2 \theta} g^{2} d v d x .
$$

For the Boltzmann equation, define the weighted dissipation norm as

$$
|g|_{\nu, \theta}^{2} \equiv \int_{\mathbf{R}^{3}} \nu(v) w^{2 \theta} g^{2} d v, \quad\|g\|_{\nu, \theta}^{2} \equiv \int_{\mathbf{R}^{3} \times \mathbf{R}^{3}} \nu(v) w^{2 \theta} g^{2} d v d x .
$$

For the Landau equation, define the weighted dissipation norm as

$$
\begin{gathered}
|g|_{\sigma, \theta}^{2}=\sum_{i, j=1}^{3} \int_{\mathbf{R}^{3}} w^{2 \theta}\left[\sigma^{i j} \partial_{i} g \partial_{j} g+\sigma^{i j} v_{i} v_{j} g^{2}\right] d v, \\
\|g\|_{\sigma, \theta}^{2}=\sum_{i, j=1}^{3} \int_{\mathbf{R}^{3}} \int_{\mathbf{R}^{3}} w^{2 \theta}\left[\sigma^{i j} \partial_{i} g \partial_{j} g+\sigma^{i j} v_{i} v_{j} g^{2}\right] d v d x,
\end{gathered}
$$

where $|\cdot|_{\sigma}=|\cdot|_{\sigma, 0},\|\cdot\|_{\sigma}=\|\cdot\|_{\sigma, 0}$ and $\sigma^{i j}$ is defined as

$$
\sigma^{i j}=\int_{\mathbf{R}^{3}} \phi^{i j}\left(v-v^{\prime}\right) \mu\left(v^{\prime}\right) d v^{\prime} .
$$


We will unify the notation as $\|g\|_{w, \theta}$, which denotes either $\|g\|_{\nu, \theta}$ or $\|g\|_{\sigma, \theta}$ and if $\theta=0$, $\|g\|_{w, 0}=\|g\|_{w}$, which denotes either $\|g\|_{\nu}$ or $\|g\|_{\sigma}$. Let $\alpha$ and $\beta$ be $\alpha=\left[\alpha_{0}, \alpha_{1}, \alpha_{2}, \alpha_{3}\right]$ and $\beta=\left[\beta_{1}, \beta_{2}, \beta_{3}\right]$. We denote

$$
\partial_{\beta}^{\alpha} \equiv \partial_{t}^{\alpha_{0}} \partial_{x_{1}}^{\alpha_{1}} \partial_{x_{2}}^{\alpha_{2}} \partial_{x_{3}}^{\alpha_{3}} \partial_{v_{1}}^{\beta_{1}} \partial_{v_{2}}^{\beta_{2}} \partial_{v_{3}}^{\beta_{3}} .
$$

If each component of $\beta$ is not greater than that of $\beta_{1}^{\prime} s$, we denote it by $\beta \leq \beta_{1}$. We define $\beta<\beta_{1}$ if $\beta \leq \beta_{1}$, and $|\beta|<\left|\beta_{1}\right|$. We denote $\left(\frac{\beta}{\beta}\right)$ by $C_{\bar{\beta}}^{\beta}$. Let

$$
\begin{gathered}
\||f|\|_{|\beta|}^{2}=\sum_{|\alpha|+|\beta| \leq N}\left\|w^{|\beta|} \partial_{\beta}^{\alpha} f(t)\right\|^{2}, \\
\left\||| f||_{w,|\beta|}^{2}=\sum_{|\beta| \leq N}\right\| w^{|\beta|} \partial_{\beta}\{\mathbf{I}-\mathbf{P}\} f\left\|_{w}^{2}+\sum_{|\alpha|+|\beta| \leq N,|\alpha| \neq 0}\right\| w^{|\beta|} \partial_{\beta}^{\alpha} f(t) \|_{w}^{2} .
\end{gathered}
$$

We next define the high order energy norm as

$$
\mathcal{E}(f(t)) \equiv \frac{1}{2}|||f(t)|\left\|_{|\beta|}^{2}+\int_{0}^{t}|||f(s)|\right\|_{w,|\beta|}^{2} d s,
$$

with the initial energy

$$
\mathcal{E}\left(f_{0}\right)=\mathcal{E}(f(0)) \equiv \sum_{|\alpha|+|\beta| \leq N}\left\|w^{|\beta|} \partial_{\beta}^{\alpha} f_{0}\right\|^{2} .
$$

Throughout this paper, $N \geq 8$. The main results are stated as follows:

Theorem 1.1. Let $F_{0}(x, v)=\mu+\sqrt{\mu} f_{0}(x, v) \geq 0$. There exist $C_{0}>0$ and $M>0$ such that if $\mathcal{E}\left(f_{0}\right) \leq M$, then there exists a unique global classical solution $f(t, x, v)$ to (1.3). Moreover, $F(t, x, v)=\mu+\sqrt{\mu} f(t, x, v) \geq 0$ solves equation (1.1) and $\sup _{0 \leq s \leq \infty} \mathcal{E}(f(s)) \leq$ $C_{0} \mathcal{E}\left(f_{0}\right)$.

Although there is extensive mathematical literature for the Boltzmann theory (see $[1,4,8,9,15,21,31,27,28,35]$ and their references), much less is known for soft potentials $\gamma<0$. Global smooth small-amplitude solutions of the Vlasov-Poisson-Boltzmann system near vacuum were constructed with $-3 \leq \gamma<-2$ in [11]. Caflisch [4] obtained the global solutions of the Boltzmann equation near Maxwellians with $-1<\gamma<0$ in a periodic box by use of spectral analysis. Recently Guo [15] generalized the results in [4] to the cases $-3<\gamma<0$ by an energy method developed in [13, 14. In the whole space, it was Ukai and Asano 28] who obtained the global solutions of the Boltzmann equation near Maxwellians with $-1<\gamma<0$. However, it is still an open problem to extend the results in 28 to the general cases. On the other hand, there have been some investigations on the global weak solutions for the inverse power law [8, 19, some even without any angular cutoff [1, 2, 29].

Despite its physical significance for the Landau equation, few global solutions have been constructed. Desvillettes and Villani [7 proved global existence and uniqueness of classical solutions for the spatially homogeneous Landau equation for hard potentials and a large class of initial data. Degond and Lemou [6] studied the spectral properties and dispersion relation of the linearized Landau operator. For the Coulomb interaction with $\gamma=-3$, global weak solutions have been studied in [2, 29, 30, up to some defect measures, while Guo [12] constructed global classical solutions near Maxwellians for a 
general Landau equation (both hard and soft potentials) in a periodic box via an energy method. There are many other studies on the Landau equation (see [28-32]).

In the present paper, we obtain global classical solutions of the Boltzmann and Landau equations near Maxwellians in the whole space. Our results generalize the classical results of Ukai and Asano [28] about the global solutions of the Boltzmann equation in the whole space to the very soft potentials $-3<\gamma<0$. On the other hand, our results extend the classical results of Claflisch [4] and Guo [15] about the Boltzmann equation in the periodic box to the whole space. For the Landau equation in the Coulomb interaction $\gamma=-3$, global weak solutions have been studied in [20, 29] and global classical solutions have recently been constructed in the periodic box in [12, while no global classical solutions in whole space have been known. Therefore, our global existence results are new on the Landau equation near Maxwellians in the whole space.

Since the solutions to the linearized Boltzmann equation around $\mu$ have a slow time decay rate for $\gamma$ near -3 , which is impossible to be used in the nonlinear problem, instead of studying the linear problem like [4, 27, 28, we directly investigate the energy estimates for the nonlinear problem. Our construction of the global solution, almost positive definite for the solution to (1.3), is based on a recent nonlinear energy method developed in [14, 16, 21, with a new a priori estimate for the linearized collision operator $L$ itself, not its time integration [13, 15, 12.

Although it has the same framework as [14, 16, 17, there are several major new difficulties in this paper. First, it is impossible to directly control $v$-derivatives $\partial_{\beta}$ of linear streaming term $v_{j} \partial^{j} f$ in terms of the weaker $\left\|\partial_{\beta} f\right\|_{w}$. Thus we introduce the additional weighted function $w$ in the energy norm like [12, 15] to overcome this difficulty. Since $\gamma<0$, the estimates of the nonlinear term $\Gamma\left(g_{1}, g_{2}\right)$ in terms of the weighted norms for $g_{1}$ and $g_{2}$ are particularly delicate. To get rid of the singularity of $\Gamma(f, f)$, we first decompose $\Gamma(f, f)$ into

$$
\Gamma(\mathbf{P} f, \mathbf{P} f)+\Gamma(\{\mathbf{I}-\mathbf{P}\} f, \mathbf{P} f)+\Gamma(\mathbf{P} f,\{\mathbf{I}-\mathbf{P}\} f)+\Gamma(\{\mathbf{I}-\mathbf{P}\} f,\{\mathbf{I}-\mathbf{P}\} f),
$$

carefully estimate each part by using the exponential decay of the hydrodynamic part $\mathbf{P} f$ about the velocity variable $v$ and we obtain the new estimates (Theorem 2.1 and Theorem 3.1), which are crucial to obtain the positivity of the linearized collision operator $L$ and the global existence of solutions. A sequence of carefully chosen decompositions of various integration regions in [12, 15] is also used to overcome the singularity in the collision kernels.

Since the Landau collision operator contains the derivative of $v$, and the estimates of the nonlinear collision term $\Gamma\left(g_{1}, g_{2}\right)$ indeed need the differentiability of $v$, we are forced to take the $v$-derivative of (1.3). Unfortunately, the zero-th order hydrodynamic part $\left\|\partial_{\beta} \mathbf{P} f\right\|_{\sigma}$ appears, which is difficult to be controlled. Therefore, we first introduce the weighted norm $\left.|||f|\right|_{w,|\beta|}$ which only excludes the $L^{2}\left(\mathbf{R}^{3}\right)$ norm of the zero-th order hydrodynamic part, compared to the norm ||$|f| \|_{|\beta|}$. Then we turn equation (1.3) into a microscopic-type equation (5.4) and use the technique of [21, 32] to overcome these difficulties. On the other hand, unlike the periodic box, the crucial Poincaré inequality does not hold for the problem in the whole space and we have to refine the energy analysis to obtain our results. 
2. Boltzmann estimates. In this section, we will give some basic estimates used to obtain global existence of solutions for the Boltzmann equation. This section is mainly devoted to the estimates for the nonlinear collision term $\Gamma\left(g_{1}, g_{2}\right)$ with $g_{i}(x, v)(i=1,2)$. In (1.3), the bilinear form $\Gamma\left(g_{1}, g_{2}\right)$ in the Boltzmann equation is

$$
\begin{aligned}
\Gamma\left(g_{1}, g_{2}\right)= & \mu^{-1 / 2}(v) Q\left(\mu^{1 / 2} g_{1}, \mu^{1 / 2} g_{2}\right) \equiv \Gamma_{\text {gain }}\left(g_{1}, g_{2}\right)-\Gamma_{\text {loss }}\left(g_{1}, g_{2}\right) \\
= & \int_{\mathbf{R}^{3}} \int_{\mathbf{S}^{2}}|u-v|^{\gamma} \mu^{1 / 2}(u) g_{1}\left(u^{\prime}\right) g_{2}\left(v^{\prime}\right) B(\vartheta) d u d \omega \\
& -\left[\int_{\mathbf{R}^{3}} \int_{\mathbf{S}^{2}}|u-v|^{\gamma} \mu^{1 / 2}(u) g_{1}(u) B(\vartheta) d u d \omega\right] g_{2}(v) .
\end{aligned}
$$

Notice that the change of variables $u-v \rightarrow u$ gives

$$
\begin{aligned}
\partial_{\beta}^{\alpha} \Gamma\left(g_{1}, g_{2}\right)= & \partial_{\beta}^{\alpha}\left[\int_{\mathbf{R}^{3}} \int_{\mathbf{S}^{2}}|u|^{\gamma} \mu^{1 / 2}(u+v) g_{1}\left(v+u_{\|}\right) g_{2}\left(v+u_{\perp}\right) B(\vartheta) d u d \omega\right] \\
& -\partial_{\beta}^{\alpha}\left[\int_{\mathbf{R}^{3}} \int_{\mathbf{S}^{2}}|u|^{\gamma} \mu^{1 / 2}(u+v) g_{1}(v+u) g_{2}(v) B(\vartheta) d u d \omega\right] \\
\equiv & \sum C_{\beta}^{\beta_{0} \beta_{1} \beta_{2}} C_{\alpha}^{\alpha_{1} \alpha_{2}} \Gamma^{0}\left(\partial_{\beta_{1}}^{\alpha_{1}} g_{1}, \partial_{\beta_{2}}^{\alpha_{2}} g_{1}\right),
\end{aligned}
$$

where the summation is over $\beta_{0}+\beta_{1}+\beta_{2}=\beta$ and $\alpha_{1}+\alpha_{2}=\alpha$. Here $u_{\|}=(u \cdot \omega) \omega$ and $u_{\perp}=u-u_{\|}$. By the product rule and a reverse change of variables, we have

$$
\begin{aligned}
\Gamma^{0}\left(\partial_{\beta_{1}}^{\alpha_{1}} g_{1}, \partial_{\beta_{2}}^{\alpha_{2}} g_{2}\right)= & \int_{\mathbf{R}^{3}} \int_{\mathbf{S}^{2}}|u-v|^{\gamma} \partial_{\beta_{0}}\left[\mu^{1 / 2}(u)\right] \partial_{\beta_{1}}^{\alpha_{1}} g_{1}\left(u^{\prime}\right) \partial_{\beta_{2}}^{\alpha_{2}} g_{2}\left(v^{\prime}\right) B(\vartheta) d u d \omega \\
& -\partial_{\beta_{2}}^{\alpha_{2}} g_{2}(v) \int_{\mathbf{R}^{3}} \int_{\mathbf{S}^{2}}|u-v|^{\gamma} \partial_{\beta_{0}}\left[\mu^{1 / 2}(u)\right] \partial_{\beta_{1}}^{\alpha_{1}} g_{1}(u) B(\vartheta) d u d \omega \\
\equiv & \Gamma_{\text {gain }}^{0}\left(\partial_{\beta_{1}}^{\alpha_{1}} g_{1}, \partial_{\beta_{2}}^{\alpha_{2}} g_{2}\right)-\Gamma_{\text {loss }}^{0}\left(\partial_{\beta_{1}}^{\alpha_{1}} g_{1}, \partial_{\beta_{2}}^{\alpha_{2}} g_{2}\right) .
\end{aligned}
$$

Theorem 2.1. Recall (2.1) and let $\beta_{0}+\beta_{1}+\beta_{2}=\beta, \alpha_{1}+\alpha_{2}=\alpha$ and $|\beta| \leq \theta$. If $g_{1}(x, v)=a(x) \mu^{1 / 4}(v)$, then

$$
\left|\left(w^{2 \theta} \Gamma^{0}\left(\partial_{\beta_{1}}^{\alpha_{1}} g_{1}, \partial_{\beta_{2}}^{\alpha_{2}} g_{2}\right), \partial_{\beta}^{\alpha} g_{3}\right)\right| \leq C \int_{\mathbf{R}^{3}}\left|\partial^{\alpha_{1}} a(x)\right|\left|\partial_{\beta_{2}}^{\alpha_{2}} g_{2}(x)\right|_{\nu, \theta}\left|\partial_{\beta}^{\alpha} g_{3}(x)\right|_{\nu, \theta} d x .
$$

If $g_{2}(x, v)=a(x) \mu^{1 / 4}(v)$, then

$$
\left|\left(w^{2 \theta} \Gamma^{0}\left(\partial_{\beta_{1}}^{\alpha_{1}} g_{1}, \partial_{\beta_{2}}^{\alpha_{2}} g_{2}\right), \partial_{\beta}^{\alpha} g_{3}\right)\right| \leq C \int_{\mathbf{R}^{3}}\left|\partial^{\alpha_{2}} a(x)\right|\left|\partial_{\beta_{1}}^{\alpha_{1}} g_{1}(x)\right|_{\nu, \theta}\left|\partial_{\beta}^{\alpha} g_{3}(x)\right|_{\nu, \theta} d x .
$$

Proof. First consider the second term $\Gamma_{\text {loss }}^{0}$ in (2.2). Note that $g_{1}(x, v)=a(x) \mu^{1 / 4}(v)$. Since $\left|\partial_{\beta_{0}}\left[\mu^{1 / 2}(u)\right]\right| \leq C e^{-|u|^{2} / 4}$, then we have

$$
\begin{aligned}
& \int_{\mathbf{R}^{3}}|u-v|^{\gamma} \partial_{\beta_{0}}\left[\mu^{1 / 2}(u)\right] \partial_{\beta_{1}}^{\alpha_{1}} g_{1}(x, u) d u \\
\leq & C\left\{\int_{\mathbf{R}^{3}} e^{-|u|^{2} / 4}\left|\partial_{\beta_{1}}^{\alpha_{1}} g_{1}(x, u)\right|^{2} d u\right\}^{1 / 2}\left\{\int_{\mathbf{R}^{3}}|u-v|^{2 \gamma} e^{-|u|^{2} / 4} d u\right\}^{1 / 2} \\
\leq & C\left\{\int_{\mathbf{R}^{3}} e^{-|u|^{2} / 4}\left|\partial_{\beta_{1}} e^{-|u|^{2} / 4}\right|^{2}\left|\partial^{\alpha_{1}} a(x)\right|^{2} d u\right\}^{1 / 2} \times[1+|v|]^{\gamma} \\
\leq & C\left|\partial^{\alpha_{1}} a(x)\right| \times[1+|v|]^{\gamma} .
\end{aligned}
$$


Therefore, by $0<B(\vartheta) \leq C$, we have

$$
\begin{aligned}
& \left|\left(w^{2 \theta} \Gamma_{\text {loss }}^{0}\left(\partial_{\beta_{1}}^{\alpha_{1}} g_{1}, \partial_{\beta_{2}}^{\alpha_{2}} g_{2}\right), \partial_{\beta}^{\alpha} g_{3}\right)\right| \\
\leq & C \int_{\mathbf{R}^{3}} \int_{\mathbf{R}^{3}}[1+|v|]^{\gamma} w^{2 \theta}\left|\partial^{\alpha_{1}} a(x)\right|\left|\partial_{\beta_{2}}^{\alpha_{2}} g_{2}(x, v) \partial_{\beta}^{\alpha} g_{3}(x, v)\right| d x d v \\
\leq & C \int_{\mathbf{R}^{3}}\left|\partial^{\alpha_{1}} a(x)\right|\left|w^{\theta} \partial_{\beta_{2}}^{\alpha_{2}} g_{2}(x)\right|_{\nu}\left|w^{\theta} \partial_{\beta}^{\alpha} g_{3}(x)\right|_{\nu} d x .
\end{aligned}
$$

Then we consider the second term $\Gamma_{\text {loss }}^{0}$ in (2.3). Since $\left|\partial_{\beta_{0}}\left[\mu^{1 / 2}(u)\right]\right| \leq C e^{-|u|^{2} / 4}$, then we have

$$
\begin{aligned}
& \int_{\mathbf{R}^{3}}|u-v|^{\gamma} \partial_{\beta_{0}}\left[\mu^{1 / 2}(u)\right] \partial_{\beta_{1}}^{\alpha_{1}} g_{1}(x, u) d u \\
\leq & C\left\{\int_{\mathbf{R}^{3}} e^{-|u|^{2} / 4}\left|\partial_{\beta_{1}}^{\alpha_{1}} g_{1}(x, u)\right|^{2} d u\right\}^{1 / 2}\left\{\int_{\mathbf{R}^{3}}|u-v|^{2 \gamma} e^{-|u|^{2} / 4} d u\right\}^{1 / 2} \\
\leq & C\left|e^{-|u|^{2} / 8} \partial_{\beta_{1}}^{\alpha_{1}} g_{1}(x)\right|_{2} \times[1+|v|]^{\gamma} .
\end{aligned}
$$

Note that $g_{2}(x, v)=a(x) \mu^{1 / 4}(v)$. By $\left|\partial_{\beta_{2}}\left[\mu^{1 / 4}(v)\right]\right| \leq C e^{-|v|^{2} / 8}$, we have

$$
\begin{aligned}
& \left|\left(w^{2 \theta} \Gamma_{\text {loss }}^{0}\left(\partial_{\beta_{1}}^{\alpha_{1}} g_{1}, \partial_{\beta_{2}}^{\alpha_{2}} g_{2}\right), \partial_{\beta}^{\alpha} g_{3}\right)\right| \\
\leq & C \int_{\mathbf{R}^{3}}\left|\partial^{\alpha_{2}} a(x)\right|\left|e^{-|u|^{2} / 8} \partial_{\beta_{1}}^{\alpha_{1}} g_{1}(x)\right|_{2} d x \int_{\mathbf{R}^{3}}[1+|v|]^{\gamma} w^{2 \theta} e^{-|v|^{2} / 8}\left|\partial_{\beta}^{\alpha} g_{3}(x, v)\right| d v \\
\leq & C \int_{\mathbf{R}^{3}}\left|\partial^{\alpha_{2}} a(x)\right|\left|e^{-|u|^{2} / 8} \partial_{\beta_{1}}^{\alpha_{1}} g_{1}(x)\right|_{2}\left|w^{\theta} \partial_{\beta}^{\alpha} g_{3}(x)\right|_{\nu} d x \\
\leq & \left.C \int_{\mathbf{R}^{3}}\left|\partial^{\alpha_{2}} a(x)\right| w^{\theta} \partial_{\beta_{1}}^{\alpha_{1}} g_{1}(x)\right|_{\nu}\left|w^{\theta} \partial_{\beta}^{\alpha} g_{3}(x)\right|_{\nu} d x,
\end{aligned}
$$

where we have used $0<B(\vartheta) \leq C$ and the exponential decay of $e^{-|u|^{2} / 8}$.

The next step is to estimate the first term $\Gamma_{\text {gain }}^{0}$ in $(2.2)$. Here the $(u, v)$ integration domain is split into two parts:

$$
\{|u| \geq|v| / 2\} \cup\{|u| \leq|v| / 2\}
$$

For the first region $\{|u| \geq|v| / 2\}$, we have

$$
e^{-|u|^{2} / 4} \leq e^{-|u|^{2} / 8} e^{-|v|^{2} / 32}
$$


Then the integral of $w^{2 \theta} \Gamma_{\text {gain }}^{0}\left(\partial_{\beta_{1}}^{\alpha_{1}} g_{1}, \partial_{\beta_{2}}^{\alpha_{2}} g_{2}\right) \partial_{\beta}^{\alpha} g_{3}$ over such a region is bounded by

$$
\begin{aligned}
& \int_{|u| \geq|v| / 2} B(\vartheta)|u-v|^{\gamma} e^{-|u|^{2} / 8} e^{-|v|^{2} / 32} w^{2 \theta}\left|\partial_{\beta_{1}}^{\alpha_{1}} g_{1}\left(x, u^{\prime}\right) \partial_{\beta_{2}}^{\alpha_{2}} g_{2}\left(x, v^{\prime}\right) \partial_{\beta}^{\alpha} g_{3}(x, v)\right| d \omega d u d v \\
& \leq C\left\{\int B(\vartheta)|u-v|^{\gamma} e^{-|u|^{2} / 8} e^{-|v|^{2} / 32} w^{2 \theta}\left|\partial_{\beta_{1}}^{\alpha_{1}} g_{1}\left(x, u^{\prime}\right)\right|^{2}\left|\partial_{\beta_{2}}^{\alpha_{2}} g_{2}\left(x, v^{\prime}\right)\right|^{2} d \omega d u d v\right\}^{1 / 2} \\
& \quad \times\left\{\int B(\vartheta)|u-v|^{\gamma} e^{-|u|^{2} / 8} e^{-|v|^{2} / 32} w^{2 \theta}\left|\partial_{\beta}^{\alpha} g_{3}(x, v)\right|^{2} d \omega d u d v\right\}^{1 / 2} \\
& \leq C\left|\partial^{\alpha_{1}} a\right|\left|w^{\theta} \partial_{\beta}^{\alpha} g_{3}(x)\right|_{\nu} \\
& \quad \times\left\{\int B(\vartheta)|u-v|^{\gamma} e^{-|u|^{2} / 8} e^{-|v|^{2} / 32} e^{-\left|u^{\prime}\right|^{2} / 8} w^{2 \theta}\left|\partial_{\beta_{2}}^{\alpha_{2}} g_{2}\left(x, v^{\prime}\right)\right|^{2} d \omega d u d v\right\}^{1 / 2} \\
& \leq C\left|\partial^{\alpha_{1}} a\right|\left|w^{\theta} \partial_{\beta}^{\alpha} g_{3}(x)\right|_{\nu}\left\{\int B(\vartheta)\left|u^{\prime}-v^{\prime}\right|^{\gamma} e^{-\left|u^{\prime}\right|^{2} / 32-\left|v^{\prime}\right|^{2} / 32}\left|\partial_{\beta_{2}}^{\alpha_{2}} g_{2}\left(x, v^{\prime}\right)\right|^{2} d \omega d u d v\right\}^{1 / 2} \\
& \leq C\left|\partial^{\alpha_{1}} a\right|\left|w^{\theta} \partial_{\beta}^{\alpha} g_{3}(x)\right|_{\nu}\left\{\int B(\vartheta)|u-v|^{\gamma} e^{-|u|^{2} / 32-|v|^{2} / 32}\left|\partial_{\beta_{2}}^{\alpha_{2}} g_{2}(x, v)\right|^{2} d \omega d u d v\right\}^{1 / 2} \\
& \leq C\left|\partial^{\alpha_{1}} a\right|\left|w^{\theta} \partial_{\beta_{2}}^{\alpha_{2}} g_{2}(x)\right|_{\nu}\left|w^{\theta} \partial_{\beta}^{\alpha} g_{3}(x)\right|_{\nu},
\end{aligned}
$$

where we have used the change of variables $(u, v) \rightarrow\left(u^{\prime}, v^{\prime}\right), B(\vartheta) \leq C$ and $w^{2 \theta} \leq 1$. Similar arguments imply that if $g_{2}(x, v)=a(x) \mu^{1 / 4}(v),\left\langle w^{2 \theta} \Gamma_{\text {gain }}^{0}\left(\partial_{\beta_{1}}^{\alpha_{1}} g_{1}, \partial_{\beta_{2}}^{\alpha_{2}} g_{2}\right), \partial_{\beta}^{\alpha} g_{3}\right\rangle$ over such a region is bounded by

$$
C\left|\partial^{\alpha_{2}} a(x)\right|\left|w^{\theta} \partial_{\beta_{1}}^{\alpha_{1}} g_{1}(x)\right|_{\nu}\left|w^{\theta} \partial_{\beta}^{\alpha} g_{3}(x)\right|_{\nu}
$$

Now we turn to the gain term over $\{|u| \leq|v| / 2\}$. Since $|u| \leq|v| / 2$ implies $|v-u| \geq$ $|v|-|u| \geq|v| / 2$, we have, from $\gamma<0$, that

$$
|u-v|^{\gamma} \leq 2^{-\gamma}|v|^{\gamma} .
$$

Further assume $|v| \leq 1$; then $|u| \leq 1 / 2$ and the gain term is bounded by

$$
\begin{aligned}
& \int_{|v| \leq 1,|u| \leq|v| / 2} B(\vartheta)|u-v|^{\gamma} e^{-|u|^{2} / 4} w^{2 \theta}\left|\partial_{\beta_{1}}^{\alpha_{1}} g_{1}\left(x, u^{\prime}\right) \partial_{\beta_{2}}^{\alpha_{2}} g_{2}\left(x, v^{\prime}\right) \partial_{\beta}^{\alpha} g_{3}(x, v)\right| d \omega d u d v \\
\leq & C \int_{|v| \leq 1} w^{2 \theta}\left|\partial_{\beta}^{\alpha} g_{3}(x, v)\right| d v \\
& \times\left\{\int_{|u| \leq 1 / 2} B(\vartheta)|u-v|^{\gamma} e^{-|u|^{2} / 4}\left|\partial_{\beta_{1}}^{\alpha_{1}} g_{1}\left(x, u^{\prime}\right) \partial_{\beta_{2}}^{\alpha_{2}} g_{2}\left(x, v^{\prime}\right)\right| d \omega d u\right\} \\
\leq & C \int_{|v| \leq 1} w^{2 \theta}\left|\partial_{\beta}^{\alpha} g_{3}(x, v)\right| d v\left\{\int_{|u| \leq 1 / 2}|u-v|^{\gamma} e^{-|u|^{2} / 2} d u\right\}^{1 / 2} \\
& \times\left\{\int_{|u| \leq 1 / 2} B(\vartheta)|v|^{\gamma}\left|\partial_{\beta_{1}}^{\alpha_{1}} g_{1}\left(x, u^{\prime}\right)\right|^{2}\left|\partial_{\beta_{2}}^{\alpha_{2}} g_{2}\left(x, v^{\prime}\right)\right|^{2} d u d \omega\right\}^{1 / 2} \\
\leq & C\left\{\int_{|u| \leq 1 / 2,|v| \leq 1} B(\vartheta)|v|^{\gamma}\left|\partial_{\beta_{1}}^{\alpha_{1}} g_{1}\left(x, u^{\prime}\right)\right|^{2}\left|\partial_{\beta_{2}}^{\alpha_{2}} g_{2}\left(x, v^{\prime}\right)\right|^{2} d u d v d \omega\right\}^{1 / 2}\left|\omega^{\theta} \partial_{\beta}^{\alpha} g_{3}(x)\right|_{\nu} .
\end{aligned}
$$

We now estimate the first factor. Since $|u| \leq|v| / 2$, we have

$$
\left|u^{\prime}\right|+\left|v^{\prime}\right| \leq 2[|u|+|v|] \leq 3|v|
$$


Since $\gamma<0$, this implies that

$$
\begin{gathered}
|v|^{\gamma} \leq 3^{-\gamma}\left|u^{\prime}\right|^{\gamma}, \quad|v|^{\gamma} \leq 3^{-\gamma}\left|v^{\prime}\right|^{\gamma} . \\
\int_{|u| \leq 1 / 2,|v| \leq 1} B(\vartheta)|v|^{\gamma}\left|\partial_{\beta_{1}}^{\alpha_{1}} g_{1}\left(x, u^{\prime}\right)\right|^{2}\left|\partial_{\beta_{2}}^{\alpha_{2}} g_{2}\left(x, v^{\prime}\right)\right|^{2} d u d v d \omega \\
\leq C \int_{\left|u^{\prime}\right| \leq 3,\left|v^{\prime}\right| \leq 3} B(\vartheta) \min \left[\left|u^{\prime}\right|^{\gamma},\left|v^{\prime}\right|^{\gamma}\right]\left|\partial_{\beta_{1}}^{\alpha_{1}} g_{1}\left(x, u^{\prime}\right)\right|^{2}\left|\partial_{\beta_{2}}^{\alpha_{2}} g_{2}\left(x, v^{\prime}\right)\right|^{2} d u d v d \omega .
\end{gathered}
$$

Now change variables $(v, u) \rightarrow\left(v^{\prime}, u^{\prime}\right)$ so that the above is bounded by

$$
\begin{aligned}
& C \int_{|u| \leq 3,|v| \leq 3} B(\vartheta) \min \left[|u|^{\gamma},|v|^{\gamma}\right] e^{-|u|^{2} / 8}\left|\partial^{\alpha_{1}} a(x)\right|^{2}\left|\partial_{\beta_{2}}^{\alpha_{2}} g_{2}(x, v)\right|^{2} d u d v d \omega \\
& \quad \leq C\left|\partial^{\alpha_{1}} a(x)\right|^{2} \int_{|v| \leq 3}\left|\partial_{\beta_{2}}^{\alpha_{2}} g_{2}(x, v)\right|^{2} d v \leq C\left|\partial^{\alpha_{1}} a(x)\right|^{2}\left|w^{\theta} \partial_{\beta_{2}}^{\alpha_{2}} g_{2}(x)\right|_{\nu}^{2} .
\end{aligned}
$$

Thus, $\left\langle w^{2 \theta} \Gamma_{\text {gain }}^{0}\left(\partial_{\beta_{1}}^{\alpha_{1}} g_{1}, \partial_{\beta_{2}}^{\alpha_{2}} g_{2}\right), \partial_{\beta}^{\alpha} g_{3}\right\rangle$ over $\{|u| \leq|v| / 2,|v| \leq 1\}$ is bounded by

$$
C\left|\partial^{\alpha_{1}} a(x)\right|\left|w^{\theta} \partial_{\beta_{2}}^{\alpha_{2}} g_{2}(x)\right|_{\nu}\left|\omega^{\theta} \partial_{\beta}^{\alpha} g_{3}(x)\right|_{\nu}
$$

If $g_{2}(x, v)=a(x) \mu^{1 / 4}(v)$, by similar arguments, $\left\langle w^{2 \theta} \Gamma_{\text {gain }}^{0}\left(\partial_{\beta_{1}}^{\alpha_{1}} g_{1}, \partial_{\beta_{2}}^{\alpha_{2}} g_{2}\right), \partial_{\beta}^{\alpha} g_{3}\right\rangle$ over such a region is bounded by

$$
C\left|\partial^{\alpha_{2}} a(x)\right|\left|w^{\theta} \partial_{\beta_{1}}^{\alpha_{1}} g_{1}(x)\right|_{\nu}\left|w^{\theta} \partial_{\beta}^{\alpha} g_{3}(x)\right|_{\nu}
$$

The last case is the gain term over the region $\{|u| \leq|v| / 2,|v| \geq 1\}$. The integral of $\left\langle w^{2 \theta} \Gamma_{\text {gain }}^{0}\left(\partial_{\beta_{1}}^{\alpha_{1}} g_{1}, \partial_{\beta_{2}}^{\alpha_{2}} g_{2}\right), \partial_{\beta}^{\alpha} g_{3}\right\rangle$ over such a region is bounded by

$$
\begin{aligned}
& \int_{|v| \geq 1,|u| \leq|v| / 2} B(\vartheta)|u-v|^{\gamma} e^{-|u|^{2} / 4} w^{2 \theta}\left|\partial_{\beta_{1}}^{\alpha_{1}} g_{1}\left(x, u^{\prime}\right) \partial_{\beta_{2}}^{\alpha_{2}} g_{2}\left(x, v^{\prime}\right) \partial_{\beta}^{\alpha} g_{3}(x, v)\right| d \omega d u d v \\
& \leq C\left\{\int_{|v| \geq 1,|u| \leq|v| / 2} B(\vartheta)[1+|v|]^{\gamma} e^{-|u|^{2} / 4} w^{2 \theta}\left|\partial_{\beta_{1}}^{\alpha_{1}} g_{1}\left(x, u^{\prime}\right)\right|^{2}\left|\partial_{\beta_{2}}^{\alpha_{2}} g_{2}\left(x, v^{\prime}\right)\right|^{2} d u d v d \omega\right\}^{1 / 2} \\
& \quad \times\left\{\int_{|v| \geq 1,|u| \leq|v| / 2} B(\vartheta)[1+|v|]^{\gamma} e^{-|u|^{2} / 4} w^{2 \theta}\left|\partial_{\beta}^{\alpha} g_{3}(x, v)\right|^{2} d \omega d u d v\right\}^{1 / 2} .
\end{aligned}
$$

Here we have used $|u-v|^{\gamma} \leq 4^{-\gamma}[1+|v|]^{\gamma}$ in the above inequality.

Recalling (2.4), $w^{2 \theta}(v) \leq C \min \left[w^{2 \theta}\left(u^{\prime}\right), w^{2 \theta}\left(v^{\prime}\right)\right]$ for $\theta \geq 0$. Using the change of variables $(v, u) \rightarrow\left(v^{\prime}, u^{\prime}\right)$, the first factor is bounded by

$$
\begin{aligned}
& \int_{|v| \geq 1,|u| \leq|v| / 2} B(\vartheta)[1+|v|]^{\gamma} e^{-|u|^{2} / 4} w^{2 \theta}\left|\partial_{\beta_{1}}^{\alpha_{1}} g_{1}\left(x, u^{\prime}\right)\right|^{2}\left|\partial_{\beta_{2}}^{\alpha_{2}} g_{2}\left(x, v^{\prime}\right)\right|^{2} d u d v d \omega \\
\leq & C \int B(\vartheta)\left[1+\left|v^{\prime}\right|\right]^{\gamma} \min \left[w^{2 \theta}\left(u^{\prime}\right), w^{2 \theta}\left(v^{\prime}\right)\right]\left|\partial_{\beta_{1}}^{\alpha_{1}} g_{1}\left(x, u^{\prime}\right)\right|^{2}\left|\partial_{\beta_{2}}^{\alpha_{2}} g_{2}\left(x, v^{\prime}\right)\right|^{2} d u d v d \omega \\
\leq & C \int B(\vartheta)[1+|v|]^{\gamma} \min \left[w^{2 \theta}(u), w^{2 \theta}(v)\right]\left|\partial_{\beta_{1}}^{\alpha_{1}} g_{1}(x, u)\right|^{2}\left|\partial_{\beta_{2}}^{\alpha_{2}} g_{2}(x, v)\right|^{2} d u d v d \omega \\
\leq & C\left|\partial^{\alpha_{1}} a(x)\right|^{2}\left|w^{\theta} \partial_{\beta_{2}}^{\alpha_{2}} g_{2}\right|_{\nu}^{2} .
\end{aligned}
$$

Therefore, the integral of $\left\langle w^{2 \theta} \Gamma_{\text {gain }}^{0}\left[\partial_{\beta_{1}}^{\alpha_{1}} g_{1}, \partial_{\beta_{2}}^{\alpha_{2}} g_{2}\right], \partial_{\beta}^{\alpha} g_{3}\right\rangle$ over $\{|u| \leq|v| / 2,|v| \geq 1\}$ is bounded by

$$
C\left|\partial^{\alpha_{1}} a(x)\right|\left|w^{\theta} \partial_{\beta_{2}}^{\alpha_{2}} g_{2}\right|_{\nu}\left|w^{\theta} \partial_{\beta}^{\alpha} g_{3}\right|_{\nu}
$$


If $g_{2}(x, v)=a(x) \mu^{1 / 4}(v)$, by similar arguments, $\left\langle w^{2 \theta} \Gamma_{\text {gain }}^{0}\left(\partial_{\beta_{1}}^{\alpha_{1}} g_{1}, \partial_{\beta_{2}}^{\alpha_{2}} g_{2}\right), \partial_{\beta}^{\alpha} g_{3}\right\rangle$ over such a region is bounded by

$$
C\left|\partial^{\alpha_{2}} a\right|\left|w^{\theta} \partial_{\beta_{1}}^{\alpha_{1}} g_{1}(x)\right|_{\nu}\left|w^{\theta} \partial_{\beta}^{\alpha} g_{3}(x)\right|_{\nu}
$$

We deduce (2.2) and (2.3) by further integrating over $\mathbf{R}^{3}$ about the $x$ variable.

Corollary 2.2. Let $|\alpha|+|\beta| \leq N$ and $|\beta| \leq \theta$. If $g_{1}(x, v)$ belongs to the null space $N$ of $L$, namely, $g_{1}(x, v)=a(x) \sqrt{\mu}+\sum_{j=1}^{3} b_{j}(x) v_{j} \sqrt{\mu}+c(x)|v|^{2} \sqrt{\mu}$, then

$$
\begin{array}{r}
\left|\left(w^{2 \theta} \partial_{\beta}^{\alpha} \Gamma\left(g_{1}, g_{2}\right), \partial_{\beta}^{\alpha} g_{3}\right)\right| \leq C \sum \int_{\mathbf{R}^{3}}\left[\left|\partial^{\alpha_{1}} a(x)\right|+\left|\partial^{\alpha_{1}} b_{j}(x)\right|+\left|\partial^{\alpha_{1}} c(x)\right|\right] \\
\times\left|\partial_{\beta-\beta_{1}}^{\alpha-\alpha_{1}} g_{2}(x)\right|_{\nu, \theta}\left|\partial_{\beta}^{\alpha} g_{3}(x)\right|_{\nu, \theta} d x
\end{array}
$$

and if $g_{2}(x, v)=a(x) \sqrt{\mu}+\sum_{j=1}^{3} b_{j}(x) v_{j} \sqrt{\mu}+c(x)|v|^{2} \sqrt{\mu}$, then

$$
\begin{aligned}
\left|\left(w^{2 \theta} \partial_{\beta}^{\alpha} \Gamma\left(g_{1}, g_{2}\right), \partial_{\beta}^{\alpha} g_{3}\right)\right| \leq C \sum \int_{\mathbf{R}^{3}}\left[\left|\partial^{\alpha-\alpha_{1}} a(x)\right|+\left|\partial^{\alpha-\alpha_{1}} b_{j}(x)\right|+\left|\partial^{\alpha-\alpha_{1}} c(x)\right|\right] \\
\times\left|\partial_{\beta_{1}}^{\alpha_{1}} g_{1}(x)\right|_{\nu, \theta}\left|\partial_{\beta}^{\alpha} g_{3}(x)\right|_{\nu, \theta} d x
\end{aligned}
$$

where the summation is over $j,\left|\alpha_{1}\right|+\left|\beta_{1}\right| \leq N$ and $\beta_{1} \leq \beta$.

Lemma 2.3. Let $0<\left|\alpha_{1}\right|+\left|\alpha_{2}\right|=|\alpha| \leq N$ and $h(v)$ be a smooth function so that $|h(v)|+|\nabla h(v)|+\left|\nabla^{2} h(v)\right| \leq C \mu^{1 / 4}(v)$; then we have

$$
\begin{aligned}
\left|\left\langle\Gamma\left(g_{1}, g_{2}\right), h\right\rangle\right|_{2} \leq\left\{\begin{array}{l}
C\left[\sum_{\left|\alpha_{i}\right|+\left|\beta_{i}\right| \leq N}\left\|w^{\left|\beta_{i}\right|} \partial_{\beta_{i}}^{\alpha_{i}} g_{1}\right\|\right]\left\|g_{2}\right\|_{\nu}, \\
C\left[\sum_{\left|\alpha_{i}\right|+\left|\beta_{i}\right| \leq N}\left\|w^{\left|\beta_{i}\right|} \partial_{\beta_{i}}^{\alpha_{i}} g_{2}\right\|\right]\left\|g_{1}\right\|_{\nu},
\end{array}\right. \\
\leq\left\{\begin{array}{l}
\left|\left\langle\Gamma\left(\partial^{\alpha_{1}} g_{1}, \partial^{\alpha_{2}} g_{2}\right), h\right\rangle\right|_{2} \\
C\left[\sum_{\left|\alpha_{i}\right|+\left|\beta_{i}\right| \leq N}\left\|w^{\left|\beta_{i}\right|} \partial_{\beta_{i}}^{\alpha_{i}} g_{1}\right\|\right]\left\|\partial^{\alpha_{2}} g_{2}\right\|_{\nu}, \text { if }\left|\alpha_{1}\right| \leq \frac{N}{2} ; \\
C\left[\sum_{\left|\alpha_{i}\right|+\left|\beta_{i}\right| \leq N}\left\|w^{\left|\beta_{i}\right|} \partial_{\beta_{i}}^{\alpha_{i}} g_{2}\right\|\right]\left\|\partial^{\alpha_{1}} g_{1}\right\|_{\nu}, \text { if }\left|\alpha_{1}\right|>\frac{N}{2}
\end{array}\right.
\end{aligned}
$$

Proof. Notice that for the Boltzmann equation,

$$
\begin{aligned}
\Gamma\left(g_{1}, g_{2}\right)= & \int_{\mathbf{R}^{3} \times \mathbf{S}^{2}}|u-v|^{\gamma} \mu^{1 / 2}(u) g_{1}\left(u^{\prime}\right) g_{2}\left(v^{\prime}\right) B(\vartheta) d u d \omega \\
& \quad-\left[\int_{\mathbf{R}^{3} \times \mathbf{S}^{2}}|u-v|^{\gamma} \mu^{1 / 2}(u) g_{1}(u) B(\vartheta) d u d \omega\right] g_{2}(v) \\
\equiv & \Gamma_{\text {gain }}^{0}\left(g_{1}, g_{2}\right)-\Gamma_{\text {loss }}^{0}\left(g_{1}, g_{2}\right) .
\end{aligned}
$$


We first estimate the loss term $\Gamma_{\text {loss }}\left(g_{1}, g_{2}\right)$. The term $\Gamma_{\text {loss }}\left(g_{1}, g_{2}\right)$ is estimated as

$$
\begin{aligned}
& \left|\left\langle\Gamma_{\text {loss }}\left(g_{1}, g_{2}\right), h\right\rangle\right| \\
\leq & C \int_{\mathbf{R}^{3}}\left|g_{2}(v) h(v)\right| \int_{\mathbf{R}^{3}}|u-v|^{\gamma} e^{-|u|^{2} / 2}\left|g_{1}(u)\right| d u d v \\
\leq & C \int_{\mathbf{R}^{3}}\left|g_{2}(v) h(v)\right| d v\left\{\int_{\mathbf{R}^{3}}|u-v|^{2 \gamma} e^{-|u|^{2} / 2} d u\right\}^{1 / 2}\left\{\int_{\mathbf{R}^{3}} e^{-|u|^{2} / 2}\left|g_{1}(u)\right|^{2} d u\right\}^{1 / 2} \\
\leq & C\left\{\int_{\mathbf{R}^{3}} e^{-|u|^{2} / 2}\left|g_{1}(u)\right|^{2} d u\right\}^{1 / 2} \int_{\mathbf{R}^{3}}[1+|v|]^{\gamma}\left|g_{2}(v)\right| e^{-|v|^{2} / 4} d v \\
\leq & C\left\{\int_{\mathbf{R}^{3}} e^{-|u|^{2} / 2}\left|g_{1}(u)\right|^{2} d u\right\}^{1 / 2}\left\{\int_{\mathbf{R}^{3}} e^{-|v|^{2} / 4}\left|g_{2}(v)\right|^{2} d v\right\}^{1 / 2}
\end{aligned}
$$

where we have used the Cauchy-Schwartz inequality and $h(v) \leq C e^{-|v|^{2} / 4}$.

By $H^{4}\left(\mathbf{R}^{3} \times \mathbf{R}^{3}\right) \subset L^{\infty}\left(\mathbf{R}^{3} \times \mathbf{R}^{3}\right)$, it follows that

$$
\sup _{x, u}\left\{\left.\left|e^{-|u|^{2} / 8}\right| g_{1}(x, u)\right|^{2}\right\} \leq C \sum_{\left|\alpha_{i}\right|+\left|\beta_{i}\right| \leq N}\left\|w^{\left|\beta_{i}\right|} \partial_{\beta_{i}}^{\alpha_{i}} g_{1}\right\|^{2} .
$$

Using the exponential factor, integrating over $u$ and taking the $L^{2}$ norm in $x$ for the last factor of (2.9) yields the first inequality of $\Gamma_{\text {loss }}\left(g_{1}, g_{2}\right)$ of (2.7). By a similar argument, we can obtain the second inequality of $\Gamma_{\text {loss }}\left(g_{1}, g_{2}\right)$ of $(2.7)$.

For the gain term, since $|h(v)| \leq C e^{-|v|^{2} / 4}$ and $0<B(\vartheta) \leq C$, we have

$$
\begin{aligned}
& \left|\left\langle\Gamma_{\text {gain }}\left(g_{1}, g_{2}\right), h\right\rangle\right| \\
\leq & C \int_{\mathbf{R}^{3}} \int_{\mathbf{R}^{3}} \int_{\mathbf{S}^{2}} B(\vartheta)|u-v|^{\gamma}\left|g_{1}\left(u^{\prime}\right)\right|\left|g_{2}\left(v^{\prime}\right) h(v)\right| e^{-|u|^{2} / 2} d u d v d \omega \\
\leq & C \int_{\mathbf{R}^{3}} \int_{\mathbf{S}^{2}}\left\{\int_{\mathbf{R}^{3}}|u-v|^{\gamma} e^{-|u|^{2} / 2}\left|g_{1}\left(u^{\prime}\right)\right|^{2}\left|g_{1}\left(v^{\prime}\right)\right|^{2} d u\right\}^{1 / 2} \\
& \times\left\{\int_{\mathbf{R}^{3}}|u-v|^{\gamma} e^{-|u|^{2} / 2} d u\right\}^{1 / 2} B(\vartheta)|h(v)| d v d \omega \\
\leq & C\left\{\int_{\mathbf{R}^{3}} \int_{\mathbf{R}^{3}} \int_{\mathbf{S}^{2}} B(\vartheta)|u-v|^{\gamma} e^{-|u|^{2} / 2}|h(v)|\left|g_{1}\left(u^{\prime}\right)\right|^{2}\left|g_{2}\left(v^{\prime}\right)\right|^{2} d u d v d \omega\right\}^{1 / 2} \\
& \times\left\{\int_{\mathbf{R}^{3}} \int_{\mathbf{S}^{2}} B(\vartheta)|h(v)|[1+|v|]^{\gamma} d v d \omega\right\}^{1 / 2} \\
\leq & C\left\{\int_{\mathbf{R}^{3}} \int_{\mathbf{R}^{3}} \int_{\mathbf{S}^{2}} B(\vartheta)|u-v|^{\gamma} e^{-\left|u^{\prime}\right|^{2} / 4-\left|v^{\prime}\right|^{2} / 4}\left|g_{1}\left(u^{\prime}\right)\right|^{2}\left|g_{2}\left(v^{\prime}\right)\right|^{2} d u d v d \omega\right\}^{1 / 2} \\
\leq & C\left\{\int_{\mathbf{R}^{3}} \int_{\mathbf{R}^{3}} \int_{\mathbf{S}^{2}} B(\vartheta)|u-v|^{\gamma} e^{-|u|^{2} / 4-|v|^{2} / 4}\left|g_{1}(u)\right|^{2}\left|g_{2}(v)\right|^{2} d u d v d \omega\right\}^{1 / 2},
\end{aligned}
$$


where we have used the change of variables $\left(u^{\prime}, v^{\prime}\right) \rightarrow(u, v)$. To establish the first inequality of (2.7), we have, from the above inequality, that

$$
\begin{aligned}
\left|\left\langle\Gamma_{\text {gain }}\left(g_{1}, g_{2}\right), h\right\rangle\right| \leq & C \sup _{x, u}\left\{\left.\left|e^{-|u|^{2} / 8}\right| g_{1}(x, u)\right|^{2}\right\}^{1 / 2} \\
& \times\left\{\int_{\mathbf{R}^{3}} e^{-|v|^{2} / 4}\left|g_{2}(v)\right|^{2} d v \int_{\mathbf{R}^{3}}|u-v|^{\gamma} e^{-|u|^{2} / 8} d u\right\}^{1 / 2} \\
\leq & C \sup _{x, u}\left\{\left.\left|e^{-|u|^{2} / 8}\right| g_{1}(x, u)\right|^{2}\right\}^{1 / 2}\left\{\int_{\mathbf{R}^{3}}[1+|v|]^{\gamma}\left|g_{2}(v)\right|^{2} d v d x\right\}^{1 / 2} \\
\leq & C \sum_{\left|\alpha_{i}\right|+\left|\beta_{i}\right| \leq N}\left\|w^{\left|\beta_{i}\right|} \partial_{\beta_{i}}^{\alpha_{i}} g_{1}\right\|\left|g_{2}\right|_{\nu},
\end{aligned}
$$

where we have used (2.10). We deduce the first inequality of (2.7) by taking the $L^{2}$ norm in $x$. Similar arguments to the above imply that the second inequality of (2.7) holds. The proof of $(2.8)$ can be found in [15].

Next, we borrow two lemmas from [15] for the completeness of the paper.

Lemma 2.4. There is some constant $C>0$ such that

$$
\left|\left\langle K g_{1}, g_{2}\right\rangle\right| \leq C\left|g_{1}\right|_{\nu}\left|g_{2}\right|_{\nu} .
$$

Let $|\beta|>0$. For any $\eta>0$, there exists $C_{\eta}>0$ such that

$$
\left\langle w^{2 \theta} \partial_{\beta}[L g], \partial_{\beta} g\right\rangle \geq\left|w^{\theta} \partial_{\beta} g\right|_{\nu}^{2}-\eta \sum_{\left|\beta_{1}\right| \leq|\beta|}\left|w^{\theta} \partial_{\beta_{1}} g\right|_{\nu}^{2}-C_{\eta}\left|w^{\theta} g\right|_{\nu}^{2} .
$$

Lemma 2.5. Recall (2.1) and let $\beta_{0}+\beta_{1}+\beta_{2}=\beta, \alpha_{1}+\alpha_{2}=\alpha$ and $|\beta| \leq \theta$. If $\left|\alpha_{1}\right|+\left|\beta_{1}\right| \leq N / 2$, then

$$
\begin{aligned}
& \left|\left(w^{2 \theta} \Gamma^{0}\left(\partial_{\beta_{1}}^{\alpha_{1}} g_{1}, \partial_{\beta_{2}}^{\alpha_{2}} g_{2}\right), \partial_{\beta}^{\alpha} g_{3}\right)\right| \\
\leq & C\left[\sum_{\left|\alpha_{i}\right|+\left|\beta_{i}\right| \leq N}\left\|w^{\left|\beta_{i}\right|} \partial_{\beta_{i}}^{\alpha_{i}} g_{1}\right\|\right]\left\|w^{\theta} \partial_{\beta_{2}}^{\alpha_{2}} g_{2}\right\|_{\nu}\left\|w^{\theta} \partial_{\beta}^{\alpha} g_{3}\right\|_{\nu} \\
& +C\left[\sum_{\left|\alpha_{i}\right|+\left|\beta_{1}\right| \leq N}\left\|w^{\left|\beta_{1}\right|} \partial_{\beta_{1}}^{\alpha_{i}} g_{1}\right\|\right]\left\|w^{\theta-\left|\beta_{1}\right|} \partial_{\beta_{2}}^{\alpha_{2}} g_{2}\right\|_{\nu}\left\|w^{\theta} \partial_{\beta}^{\alpha} g_{3}\right\|_{\nu} .
\end{aligned}
$$

If $\left|\alpha_{1}\right|+\left|\beta_{1}\right| \geq N / 2$, then

$$
\begin{aligned}
& \left|\left(w^{2 \theta} \Gamma^{0}\left(\partial_{\beta_{1}}^{\alpha_{1}} g_{1}, \partial_{\beta_{2}}^{\alpha_{2}} g_{2}\right), \partial_{\beta}^{\alpha} g_{3}\right)\right| \\
\leq & C\left[\sum_{\left|\alpha_{i}\right|+\left|\beta_{i}\right| \leq N}\left\|w^{\left|\beta_{i}\right|} \partial_{\beta_{i}}^{\alpha_{i}} g_{2}\right\|\right]\left\|w^{\theta} \partial_{\beta_{1}}^{\alpha_{1}} g_{1}\right\|_{\nu}\left\|w^{\theta} \partial_{\beta}^{\alpha} g_{3}\right\|_{\nu} \\
& +C\left[\sum_{\left|\alpha_{i}\right|+\left|\beta_{2}\right| \leq N}\left\|w^{\left|\beta_{2}\right|} \partial_{\beta_{2}}^{\alpha_{i}} g_{2}\right\|\right]\left\|w^{\theta-\left|\beta_{2}\right|} \partial_{\beta_{1}}^{\alpha_{1}} g_{1}\right\|_{\nu}\left\|w^{\theta} \partial_{\beta}^{\alpha} g_{3}\right\|_{\nu} .
\end{aligned}
$$

3. Landau estimates. In this section, we will prove the basic estimates used to obtain the positivity of the linearized Landau operator and global existence of solutions for the Landau equation. In this case, we need to exercise more care in estimating the nonlinear collision term $\Gamma(f, g)$, which is crucial in the later energy analysis. 
The operators $A, K$ and $\Gamma$ from (1.3) in the Landau equation are defined in [12] as the following representations:

$$
\begin{gathered}
A g_{1}=\partial_{i}\left[\sigma^{i j} \partial_{j} g_{1}\right]-\sigma^{i j} v_{i} v_{j} g_{1}+\partial_{i}\left[v_{j} \sigma^{i j}\right] g_{1}, \\
K g_{2}=-\mu^{-1 / 2} \partial_{i}\left\{\mu\left[\phi^{i j} *\left\{\mu^{1 / 2}\left[\partial_{j} g_{2}+v_{j} g_{2}\right]\right\}\right]\right\}, \\
\Gamma\left(g_{1}, g_{2}\right)=\partial_{i}\left[\left\{\phi^{i j} *\left[\mu^{1 / 2} g_{1}\right]\right\} \partial_{j} g_{2}\right]-\left\{\phi^{i j} *\left[v_{i} \mu^{1 / 2} g_{1}\right]\right\} \partial_{j} g_{2} \\
-\partial_{i}\left[\left\{\phi^{i j} *\left[\mu^{1 / 2} \partial_{j} g_{1}\right]\right\} g_{2}\right]+\left\{\phi^{i j} *\left[v_{i} \mu^{1 / 2} \partial_{j} g_{1}\right]\right\} g_{2} .
\end{gathered}
$$

For any vector-valued function $g(v)=\left(g_{1}, g_{2}, g_{3}\right)$, we define the projection to the vector $v$ as

$$
\mathbf{P}_{v} g_{i} \equiv \frac{v_{i}}{|v|} \sum_{j=1}^{3} \frac{v_{j}}{|v|} g_{j}, \quad 1 \leq i \leq 3 .
$$

By Corollary 1 of [12, there exists some constant $c>0$ such that

$$
\left|[1+|v|]^{\frac{\gamma}{2}}\left\{\mathbf{P}_{v} \partial_{i} g\right\}\right|_{2, \theta}^{2}+\left|[1+|v|]^{\frac{\gamma+2}{2}}\left\{\left[\mathbf{I}-\mathbf{P}_{v}\right] \partial_{i} g\right\}\right|_{2, \theta}^{2}+\left|[1+|v|]^{\frac{\gamma+2}{2}} g\right|_{2, \theta}^{2} \leq c|g|_{\sigma, \theta}^{2}
$$

Theorem 3.1. Recall (3.3) and let $|\alpha|+|\beta| \leq N$ and $|\beta| \leq \theta$. If $g_{1}(x, v)=a(x) \mu^{1 / 4}(v)$, then

$$
\left|\left\langle w^{2 \theta} \partial_{\beta}^{\alpha} \Gamma\left(g_{1}, g_{2}\right), \partial_{\beta}^{\alpha} g_{3}\right\rangle\right| \leq C \sum\left|\partial^{\alpha_{1}} a(x)\right|\left|w^{\theta} \partial_{\beta-\beta_{1}}^{\alpha-\alpha_{1}} g_{2}(x)\right|_{\sigma}\left|w^{\theta} \partial_{\beta}^{\alpha} g_{3}(x)\right|_{\sigma} .
$$

If $g_{2}(x, v)=a(x) \mu^{1 / 4}(v)$, then

$$
\left|\left\langle w^{2 \theta} \partial_{\beta}^{\alpha} \Gamma\left(g_{1}, g_{2}\right), \partial_{\beta}^{\alpha} g_{3}\right\rangle\right| \leq C \sum\left|\partial^{\alpha-\alpha_{1}} a(x)\right|\left|w^{\theta} \partial_{\beta_{1}}^{\alpha_{1}} g_{1}(x)\right|_{\sigma}\left|w^{\theta} \partial_{\beta}^{\alpha} g_{3}(x)\right|_{\sigma},
$$

where the summation is over $\left|\alpha_{1}\right|+\left|\beta_{1}\right| \leq N, \beta_{1} \leq \beta$.

Proof. By the product rule, we expand

$$
\left\langle w^{2 \theta} \partial_{\beta}^{\alpha} \Gamma\left(g_{1}, g_{2}\right), \partial_{\beta}^{\alpha} g_{3}\right\rangle=\sum C_{\alpha}^{\alpha_{1}} C_{\beta}^{\beta_{1}} \times G_{\alpha_{1} \beta_{1}},
$$

where $G_{\alpha_{1} \beta_{1}}$ takes the form:

$$
\begin{aligned}
& -\left\langle w^{2 \theta}\left\{\phi^{i j} * \partial_{\beta_{1}}\left[\mu^{1 / 2} \partial^{\alpha_{1}} g_{1}\right]\right\} \partial_{j} \partial_{\beta-\beta_{1}}^{\alpha-\alpha_{1}} g_{2}, \partial_{i} \partial_{\beta}^{\alpha} g_{3}\right\rangle \\
& -\left\langle w^{2 \theta}\left\{\phi^{i j} * \partial_{\beta_{1}}\left[v_{i} \mu^{1 / 2} \partial^{\alpha_{1}} g_{1}\right]\right\} \partial_{j} \partial_{\beta-\beta_{1}}^{\alpha-\alpha_{1}} g_{2}, \partial_{\beta}^{\alpha} g_{3}\right\rangle \\
& +\left\langle w^{2 \theta}\left\{\phi^{i j} * \partial_{\beta_{1}}\left[\mu^{1 / 2} \partial_{j} \partial^{\alpha_{1}} g_{1}\right]\right\} \partial_{\beta-\beta_{1}}^{\alpha-\alpha_{1}} g_{2}, \partial_{i} \partial_{\beta}^{\alpha} g_{3}\right\rangle \\
& +\left\langle w^{2 \theta}\left\{\phi^{i j} * \partial_{\beta_{1}}\left[v_{i} \mu^{1 / 2} \partial_{j} \partial^{\alpha_{1}} g_{1}\right]\right\} \partial_{\beta-\beta_{1}}^{\alpha-\alpha_{1}} g_{2}, \partial_{\beta}^{\alpha} g_{3}\right\rangle \\
& -\left\langle\partial_{i}\left[w^{2 \theta}\right]\left\{\phi^{i j} * \partial_{\beta_{1}}\left[\mu^{1 / 2} \partial^{\alpha_{1}} g_{1}\right]\right\} \partial_{j} \partial_{\beta-\beta_{1}}^{\alpha-\alpha_{1}} g_{2}, \partial_{\beta}^{\alpha} g_{3}\right\rangle \\
& +\left\langle\partial_{i}\left[w^{2 \theta}\right]\left\{\phi^{i j} * \partial_{\beta_{1}}\left[\mu^{1 / 2} \partial_{j} \partial^{\alpha_{1}} g_{1}\right]\right\} \partial_{\beta-\beta_{1}}^{\alpha-\alpha_{1}} g_{2}, \partial_{\beta}^{\alpha} g_{3}\right\rangle .
\end{aligned}
$$

The last two terms appear when we integrate by parts over the $v_{i}$ variable. 
We first establish (3.7). Since $\phi^{i j} \in L_{l o c}^{2}\left(\mathbf{R}^{3}\right)$ and $\left|\partial_{\beta_{1}-\bar{\beta}}\left[\mu^{1 / 2}\right]\right| \leq C \mu^{1 / 4}$, we deduce that

$$
\begin{aligned}
\left\{\phi^{i j} * \partial_{\beta_{1}}\left[\mu^{1 / 2} \partial^{\alpha_{1}} g_{1}\right]\right\} & \leq C\left[\left|\phi^{i j}\right|^{2} * \mu^{1 / 4}\right]^{1 / 2} \sum_{\bar{\beta} \leq \beta_{1}}\left|\mu^{1 / 8} \partial_{\bar{\beta}}^{\alpha_{1}} g_{1}\right|_{2} \\
& \leq C[1+|v|]^{\gamma+2} \sum_{\bar{\beta} \leq \beta_{1}}\left|w^{\theta} \partial_{\bar{\beta}}^{\alpha_{1}} g_{1}\right|_{\sigma}
\end{aligned}
$$

where we have used (3.5) and the exponential decay of $\mu^{1 / 8}(v)$.

Since $g_{2}(x, v)=a(x) \mu^{1 / 4}(v),(3.8)$ is bounded by

$$
\begin{aligned}
& C \sum_{\bar{\beta} \leq \beta_{1}}\left|w^{\theta} \partial_{\bar{\beta}}^{\alpha_{1}} g_{1}\right|_{\sigma} \int w^{2 \theta}[1+|v|]^{\gamma+2}\left|\partial_{j} \partial_{\beta-\beta_{1}}^{\alpha-\alpha_{1}} g_{2} \partial_{i} \partial_{\beta}^{\alpha} g_{3}\right| d v \\
\leq & C \sum_{\bar{\beta} \leq \beta_{1}}\left|w^{\theta} \partial_{\bar{\beta}}^{\alpha_{1}} g_{1}\right|_{\sigma}\left|\partial^{\alpha-\alpha_{1}} a(x)\right|\left|w^{\theta}[1+|v|]^{\frac{\gamma+2}{2}} \mu^{1 / 16}\right|_{2}\left|w^{\theta}[1+|v|]^{\frac{\gamma+2}{2}} \mu^{1 / 16} \partial_{i} \partial_{\beta}^{\alpha} g_{3}\right|_{2} \\
\leq & C \sum_{\bar{\beta} \leq \beta_{1}}\left|\partial^{\alpha-\alpha_{1}} a(x)\right|\left|w^{\theta} \partial_{\bar{\beta}}^{\alpha_{1}} g_{1}\right|_{\sigma}\left|w^{\theta} \partial_{\beta}^{\alpha} g_{3}\right|_{\sigma}
\end{aligned}
$$

where we have used (3.5) and the fact that $\left|\partial_{j} \partial_{\beta-\beta_{1}} \mu^{1 / 4}\right| \leq C \mu^{1 / 8}$.

Since $\partial_{\beta_{1}-\bar{\beta}}\left[v_{i} \mu^{1 / 2}\right] \leq C \mu^{1 / 4}$, then we have that

$$
\left\{\phi^{i j} * \partial_{\beta_{1}}\left[v_{i} \mu^{1 / 2} \partial^{\alpha_{1}} g_{1}\right]\right\} \leq C[1+|v|]^{\gamma+2} \sum_{\bar{\beta} \leq \beta_{1}}\left|w^{\theta} \partial_{\bar{\beta}}^{\alpha_{1}} g_{1}\right|_{\sigma} .
$$

By (3.5) and the fact that $g_{2}(x, v)=a(x) \mu^{1 / 4}(v),(3.9)$ is bounded by

$$
\begin{aligned}
& C \sum_{\bar{\beta} \leq \beta_{1}}\left|w^{\theta} \partial_{\bar{\beta}}^{\alpha_{1}} g_{1}\right|_{\sigma} \int w^{2 \theta}[1+|v|]^{\gamma+2}\left|\partial_{j} \partial_{\beta-\beta_{1}}^{\alpha-\alpha_{1}} g_{2} \partial_{\beta}^{\alpha} g_{3}\right| d v \\
\leq & C \sum_{\bar{\beta} \leq \beta_{1}}\left|w^{\theta} \partial_{\bar{\beta}}^{\alpha_{1}} g_{1}\right|_{\sigma}\left|\partial^{\alpha-\alpha_{1}} a(x)\right|\left|w^{\theta}[1+|v|]^{\frac{\gamma+2}{2}} \partial_{j} \partial_{\beta-\beta_{1}} \mu^{1 / 4}\right|_{2}\left|w^{\theta}[1+|v|]^{\frac{\gamma+2}{2}} \partial_{\beta}^{\alpha} g_{3}\right|_{2} \\
\leq & C \sum_{\bar{\beta} \leq \beta_{1}}\left|\partial^{\alpha-\alpha_{1}} a(x)\right|\left|w^{\theta} \partial_{\bar{\beta}}^{\alpha_{1}} g_{1}\right|_{\sigma}\left|w^{\theta} \partial_{\beta}^{\alpha} g_{3}\right|_{\sigma} .
\end{aligned}
$$

By (3.14) and (3.16), we deduce from (3.5) that

$$
\begin{aligned}
& \left\{\phi^{i j} * \partial_{\beta_{1}}\left[\mu^{1 / 2} \partial_{j} \partial^{\alpha_{1}} g_{1}\right]\right\}+\left\{\phi^{i j} * \partial_{\beta_{1}}\left[v_{i} \mu^{1 / 2} \partial_{j} \partial^{\alpha_{1}} g_{1}\right]\right\} \\
\leq & {\left[\left|\phi^{i j}\right|^{2} * \mu^{1 / 4}\right]^{1 / 2} \sum_{\bar{\beta} \leq \beta_{1}}\left|\mu^{1 / 8} \partial_{j} \partial_{\bar{\beta}}^{\alpha_{1}} g_{1}\right|_{2} } \\
\leq & C[1+|v|]^{\gamma+2} \sum_{\bar{\beta} \leq \beta_{1}}\left|w^{\theta} \partial_{\bar{\beta}}^{\alpha_{1}} g_{1}\right|_{\sigma} .
\end{aligned}
$$


By using (3.18) and the fact that $g_{2}(x, v)=a(x) \mu^{1 / 4}(v)$, similar arguments as those used in (3.15) and (3.17) imply that (3.10) and (3.11) can be controlled by the right-hand side of (3.7).

Since $\left|\partial_{i}\left[w^{2 \theta}\right]\right| \leq C[1+|v|]^{-1} w^{2 \theta}$ and $g_{2}(x, v)=a(x) \mu^{1 / 4}(v),(3.12)$ is bounded by

$$
\begin{aligned}
& C \sum_{\bar{\beta} \leq \beta_{1}}\left|w^{\theta} \partial_{\bar{\beta}}^{\alpha_{1}} g_{1}\right|_{\sigma} \int w^{2 \theta}[1+|v|]^{\gamma+1}\left|\partial_{j} \partial_{\beta-\beta_{1}}^{\alpha-\alpha_{1}} g_{2} \partial_{\beta}^{\alpha} g_{3}\right| d v \\
\leq & C \sum_{\bar{\beta} \leq \beta_{1}}\left|w^{\theta} \partial_{\bar{\beta}}^{\alpha_{1}} g_{1}\right|_{\sigma}\left|\partial^{\alpha-\alpha_{1}} a(x)\right|\left|w^{\theta}[1+|v|]^{\frac{\gamma}{2}} \partial_{j} \partial_{\beta-\beta_{1}} \mu^{1 / 4}\right|_{2}\left|w^{\theta}[1+|v|]^{\frac{\gamma+2}{2}} \partial_{\beta}^{\alpha} g_{3}\right|_{2} \\
\leq & C \sum_{\bar{\beta} \leq \beta_{1}}\left|\partial^{\alpha-\alpha_{1}} a(x)\right|\left|w^{\theta} \partial_{\bar{\beta}}^{\alpha_{1}} g_{1}\right|_{\sigma}\left|w^{\theta} \partial_{\beta}^{\alpha} g_{3}\right|_{\sigma}
\end{aligned}
$$

where we have used (3.5) and (3.14). By similar arguments as those used in (3.19), (3.13) is controlled by the last line of (3.19).

In the following we will establish (3.6). We first estimate (3.11) and (3.13).

Since $\left|\partial_{i}\left[w^{2 \theta}\right]\right| \leq C[1+|v|]^{-1} w^{2 \theta}$ and $g_{1}(x, v)=a(x) \mu^{1 / 4}(v),(3.11)$ and (3.13) are both bounded by

$$
\begin{aligned}
& C \sum_{\bar{\beta} \leq \beta_{1}}\left|w^{\theta} \partial_{\bar{\beta}}^{\alpha_{1}} g_{1}\right|_{\sigma} \int w^{2 \theta}[1+|v|]^{\gamma+2}\left|\partial_{\beta-\beta_{1}}^{\alpha-\alpha_{1}} g_{2} \partial_{\beta}^{\alpha} g_{3}\right| d v \\
\leq & C \sum_{\bar{\beta} \leq \beta_{1}}\left|\partial^{\alpha_{1}} a(x)\right|\left|w^{\theta} \partial_{\bar{\beta}} \mu^{1 / 4}\right|_{\sigma}\left|w^{\theta}[1+|v|]^{\frac{\gamma+2}{2}} \partial_{\beta-\beta_{1}}^{\alpha-\alpha_{1}} g_{2}\right|_{2}\left|w^{\theta}[1+|v|]^{\frac{\gamma+2}{2}} \partial_{\beta}^{\alpha} g_{3}\right|_{2} \\
\leq & C \sum_{\bar{\beta} \leq \beta_{1}}\left|\partial^{\alpha_{1}} a(x)\right|\left|w^{\theta} \partial_{\beta-\beta_{1}}^{\alpha-\alpha_{1}} g_{2}\right|_{\sigma}\left|w^{\theta} \partial_{\beta}^{\alpha} g_{3}\right|_{\sigma}
\end{aligned}
$$

where we have used (3.5) and (3.18).

Since $\left|\partial_{i}\left[w^{2 \theta}\right]\right| \leq C[1+|v|]^{-1} w^{2 \theta}$ and $g_{1}(x, v)=a(x) \mu^{1 / 4}(v)$, we easily deduce, from (3.5) and (3.18), that (3.12) is bounded by

$$
\begin{aligned}
& C \sum_{\bar{\beta} \leq \beta_{1}}\left|w^{\theta} \partial_{\bar{\beta}}^{\alpha_{1}} g_{1}\right|_{\sigma} \int w^{2 \theta}[1+|v|]^{\gamma+1}\left|\partial_{j} \partial_{\beta-\beta_{1}}^{\alpha-\alpha_{1}} g_{2} \partial_{\beta}^{\alpha} g_{3}\right| d v \\
\leq & C \sum_{\bar{\beta} \leq \beta_{1}}\left|\partial^{\alpha_{1}} a(x)\right|\left|w^{\theta} \partial_{\bar{\beta}} \mu^{1 / 4}\right|_{\sigma}\left|w^{\theta}[1+|v|]^{\frac{\gamma}{2}} \partial_{j} \partial_{\beta-\beta_{1}}^{\alpha-\alpha_{1}} g_{2}\right|_{2}\left|w^{\theta}[1+|v|]^{\frac{\gamma+2}{2}} \partial_{\beta}^{\alpha} g_{3}\right|_{2} \\
\leq & C \sum_{\bar{\beta} \leq \beta_{1}}\left|\partial^{\alpha_{1}} a(x)\right|\left|w^{\theta} \partial_{\beta-\beta_{1}}^{\alpha-\alpha_{1}} g_{2}\right|_{\sigma}\left|w^{\theta} \partial_{\beta}^{\alpha} g_{3}\right|_{\sigma}
\end{aligned}
$$

We now estimate (3.8) to (3.10). We decompose their double integration region $\left[v, v^{\prime}\right] \in$ $\mathbf{R}^{3} \times \mathbf{R}^{3}$ into three parts:

$$
\{|v| \leq 1\}, \quad\left\{2\left|v^{\prime}\right| \geq|v|,|v| \geq 1\right\} \text { and }\left\{2\left|v^{\prime}\right| \leq|v|,|v| \geq 1\right\} .
$$


For the first part, $\{|v| \leq 1\}$, recall that $\phi^{i j}(v)=O\left(|v|^{\gamma+2}\right) \in L_{l o c}^{2}$. By (3.14), (3.16) and (3.18), we have

$$
\begin{aligned}
& \left|\phi^{i j} * \partial_{\beta_{1}}\left[\mu^{1 / 2} \partial_{j} \partial^{\alpha_{1}} g_{1}\right]\right|+\left|\phi^{i j} * \partial_{\beta_{1}}\left[v_{i} \mu^{1 / 2} \partial_{j} \partial^{\alpha_{1}} g_{1}\right]\right| \\
& \quad \leq C[1+|v|]^{\gamma+2} \sum_{\bar{\beta} \leq \beta_{1}}\left|w^{\theta} \partial_{\bar{\beta}}^{\alpha_{1}} g_{1}\right|_{\sigma}, \\
& \left|\phi^{i j} * \partial_{\beta_{1}}\left[\mu^{1 / 2} \partial^{\alpha_{1}} g_{1}\right]\right|+\left|\phi^{i j} * \partial_{\beta_{1}}\left[v_{i} \mu^{1 / 2} \partial^{\alpha_{1}} g_{1}\right]\right| \\
& \quad \leq C[1+|v|]^{\gamma+2} \sum_{\bar{\beta} \leq \beta_{1}}\left|w^{\theta} \partial_{\bar{\beta}}^{\alpha_{1}} g_{1}\right|_{\sigma} .
\end{aligned}
$$

Hence their corresponding integrands over the region $\{|v| \leq 1\}$ are bounded by

$$
C \sum\left|w^{\theta} \partial_{\bar{\beta}}^{\alpha_{1}} g_{1}\right|_{\sigma} w^{2 \theta}[1+|v|]^{\gamma+2}\left[\left|\partial_{j} \partial_{\beta-\beta_{1}}^{\alpha-\alpha_{1}} g_{2}\right|+\left|\partial_{\beta-\beta_{1}}^{\alpha-\alpha_{1}} g_{2}\right|\right]\left[\left|\partial_{i} \partial_{\beta}^{\alpha} g_{3}\right|+\left|\partial_{\beta}^{\alpha} g_{3}\right|\right] .
$$

Its $v$-integral over $\{|v| \leq 1\}$ is clearly bounded by the right-hand side of (3.6) because of $(3.5)$ and the fact that $g_{1}(x, v)=a(x) \mu^{1 / 4}(v)$. We thus conclude the first part of $\{|v| \leq 1\}$ for $(3.8)$ to $(3.10)$.

For the second part, $\left\{2\left|v^{\prime}\right| \geq|v|,|v| \geq 1\right\}$, we have

$$
\left|\partial_{\beta_{1}}\left\{\mu^{1 / 2}\left(v^{\prime}\right)\right\}\right|+\left|\partial_{\beta_{1}}\left\{v_{i}^{\prime} \mu^{1 / 2}\left(v^{\prime}\right)\right\}\right| \leq C \mu^{1 / 8}\left(v^{\prime}\right) \mu^{1 / 32}(v) .
$$

By the same types of estimates as in (3.21), the $v$-integrands in (3.8) to (3.10) are bounded by

$$
\begin{aligned}
& \mu^{1 / 32}(v) w^{2 \theta}\left|\partial_{j} \partial_{\beta-\beta_{1}}^{\alpha-\alpha_{1}} g_{2}\right|\left[\left|\partial_{i} \partial_{\beta}^{\alpha} g_{3}\right|+\left|\partial_{\beta}^{\alpha} g_{3}\right|\right] \int\left|\phi^{i j}\left(v-v^{\prime}\right) \mu^{1 / 8}\left(v^{\prime}\right) \partial_{\bar{\beta}}^{\alpha_{1}} g_{1}\left(v^{\prime}\right)\right| d v^{\prime} \\
& +\mu^{1 / 32}(v) w^{2 \theta}\left|\partial_{\beta-\beta_{1}}^{\alpha-\alpha_{1}} g_{2}\right|\left[\left|\partial_{i} \partial_{\beta}^{\alpha} g_{3}\right|+\left|\partial_{\beta}^{\alpha} g_{3}\right|\right] \int\left|\phi^{i j}\left(v-v^{\prime}\right) \mu^{1 / 8}\left(v^{\prime}\right) \partial_{j} \partial_{\bar{\beta}}^{\alpha_{1}} g_{1}\left(v^{\prime}\right)\right| d v^{\prime} \\
\leq & C\left|w^{2 \theta} \partial_{\bar{\beta}}^{\alpha_{1}} g_{1}\right|_{\sigma}[1+|v|]^{\gamma+2} \mu^{1 / 32}(v) w^{2 \theta}\left[\left|\partial_{j} \partial_{\beta-\beta_{1}}^{\alpha-\alpha_{1}} g_{2}\right|+\left|\partial_{\beta-\beta_{1}}^{\alpha-\alpha_{1}} g_{2}\right|\right]\left[\left|\partial_{i} \partial_{\beta}^{\alpha} g_{3}\right|+\left|\partial_{\beta}^{\alpha} g_{3}\right|\right] \\
\leq & C\left|\partial^{\alpha_{1}} a(x)\right|[1+|v|]^{\gamma+2} \mu^{1 / 32}(v) w^{2 \theta}\left[\left|\partial_{j} \partial_{\beta-\beta_{1}}^{\alpha-\alpha_{1}} g_{2}\right|+\left|\partial_{\beta-\beta_{1}}^{\alpha-\alpha_{1}} g_{2}\right|\right]\left[\left|\partial_{i} \partial_{\beta}^{\alpha} g_{3}\right|+\left|\partial_{\beta}^{\alpha} g_{3}\right|\right],
\end{aligned}
$$

where we have used $g_{1}(x, v)=a(x) \mu^{1 / 4}(v)$. Its $v$-integral is bounded by the right-hand side of (3.6) because of the fast decay factor $\mu^{1 / 32}(v)$.

We finally consider the third part, $\left\{2\left|v^{\prime}\right| \leq|v|,|v| \geq 1\right\}$, for which we shall estimate each term from (3.8) to (3.10).

To estimate (3.8) over this region we expand $\phi^{i j}\left(v-v^{\prime}\right)$ to get

$$
\phi^{i j}\left(v-v^{\prime}\right)=\phi^{i j}(v)-\sum_{k} \partial_{k} \phi^{i j}(v) v_{k}^{\prime}+\frac{1}{2} \sum_{k, l} \partial_{k l} \phi^{i j}(\bar{v}) v_{k}^{\prime} v_{l}^{\prime},
$$

where $\bar{v}$ is between $v$ and $v-v^{\prime}$. We plug (3.22) into the integrand of (3.8). Notice that for either fixed $i$ or $j$,

$$
\sum_{i} \phi^{i j} v_{i}=\sum_{j} \phi^{i j} v_{j}=0
$$


From (3.4) and (3.23), we can decompose $\partial_{j} \partial_{\beta-\beta_{1}}^{\alpha-\alpha_{1}} g_{2}$ and $\partial_{i} \partial_{\beta}^{\alpha} g_{3}$ into $\mathbf{P}_{v}$ parts as well as $\mathbf{I}-\mathbf{P}_{v}$ parts. For the first term in expansion (3.22), we have

$$
\begin{gathered}
\sum_{i j} w^{2 \theta} \phi^{i j}(v) \partial_{j} \partial_{\beta-\beta_{1}}^{\alpha-\alpha_{1}} g_{2} \partial_{i} \partial_{\beta-\beta_{1}}^{\alpha-\alpha_{1}} g_{3} \\
=\sum_{i j} w^{2 \theta} \phi^{i j}(v)\left\{\left[\mathbf{I}-\mathbf{P}_{v}\right] \partial_{j} \partial_{\beta-\beta_{1}}^{\alpha-\alpha_{1}} g_{2}\left[\mathbf{I}-\mathbf{P}_{v}\right] \partial_{i} \partial_{\beta}^{\alpha} g_{3}\right\} .
\end{gathered}
$$

Here we have used (3.23) so that the sum of terms with either $\mathbf{P}_{v} \partial_{j} \partial_{\beta-\beta_{1}}^{\alpha-\alpha_{1}} g_{2}$ or $\mathbf{P}_{v} \partial_{i} \partial_{\beta}^{\alpha} g_{3}$ vanishes. The absolute value of this is bounded by

$$
C w^{2 \theta}[1+|v|]^{\gamma+2}\left|\left[\mathbf{I}-\mathbf{P}_{v}\right] \partial_{j} \partial_{\beta-\beta_{1}}^{\alpha-\alpha_{1}} g_{2}\right| \times\left|\left[\mathbf{I}-\mathbf{P}_{v}\right] \partial_{i} \partial_{\beta}^{\alpha} g_{3}\right| .
$$

For the second term in expansion (3.22), by taking the $k$ derivative of

$$
\sum_{i j} \phi^{i j}(v) v_{i} v_{j}=0
$$

we have that

$$
\sum_{i j} \partial_{k} \phi^{i j}(v) v_{i} v_{j}=-2 \sum_{j} \phi^{k j}(v) v_{j}=0 .
$$

Therefore, expanding $\partial_{j} \partial_{\beta-\beta_{1}}^{\alpha-\alpha_{1}} g_{2}$ and $\partial_{i} \partial_{\beta}^{\alpha} g_{3}$ into their $\mathbf{P}_{v}$ and $\mathbf{I}-\mathbf{P}_{v}$ parts yields

$$
\begin{gathered}
\sum_{i j} w^{2 \theta} \partial_{k} \phi^{i j}(v) \partial_{j} \partial_{\beta-\beta_{1}}^{\alpha-\alpha_{1}} g_{2}(v) \partial_{i} \partial_{\beta}^{\alpha} g_{3}(v) \\
=\sum_{i j} w^{2 \theta} \partial_{k} \phi^{i j}(v)\left\{\left[\mathbf{P}_{v} \partial_{j} \partial_{\beta-\beta_{1}}^{\alpha-\alpha_{1}} g_{2}\right]\left[\mathbf{I}-\mathbf{P}_{v}\right] \partial_{i} \partial_{\beta}^{\alpha} g_{3}+w^{2 \theta}\left[\mathbf{I}-\mathbf{P}_{v}\right] \partial_{j} \partial_{\beta-\beta_{1}}^{\alpha-\alpha_{1}} g_{2}\left[\mathbf{P}_{v} \partial_{i} \partial_{\beta}^{\alpha} g_{3}\right]\right\} \\
+\sum_{i j} w^{2 \theta} \partial_{k} \phi^{i j}(v)\left[\mathbf{I}-\mathbf{P}_{v}\right] \partial_{j} \partial_{\beta-\beta_{1}}^{\alpha-\alpha_{1}} g_{2}\left[\mathbf{I}-\mathbf{P}_{v}\right] \partial_{i} \partial_{\beta}^{\alpha} g_{3},
\end{gathered}
$$

where

$$
\sum_{i j} w^{2 \theta} \partial_{k} \phi^{i j}(v)\left[\mathbf{P}_{v} \partial_{j} \partial_{\beta-\beta_{1}}^{\alpha-\alpha_{1}} g_{2}\right]\left[\mathbf{P}_{v} \partial_{i} \partial_{\beta}^{\alpha} g_{3}\right]=0 .
$$

Noting that $\left|\partial_{k} \phi^{i j}(v)\right| \leq C[1+|v|]^{\gamma+1}$ for $|v| \geq 1$, we majorize the above by

$$
\begin{gathered}
C w^{\theta}[1+|v|]^{\gamma / 2}\left\{\left|\mathbf{P}_{v} \partial_{j} \partial_{\beta-\beta_{1}}^{\alpha-\alpha_{1}} g_{2}\right|+\left|\mathbf{P}_{v} \partial_{j} \partial_{\beta}^{\alpha} g_{3}\right|\right\} \\
\times C w^{\theta}[1+|v|]^{(\gamma+2) / 2}\left\{\left|\left[\mathbf{I}-\mathbf{P}_{v}\right] \partial_{j} \partial_{\beta-\beta_{1}}^{\alpha-\alpha_{1}} g_{2}\right|+\left|\left[\mathbf{I}-\mathbf{P}_{v}\right] \partial_{i} \partial_{\beta}^{\alpha} g_{3}\right|\right\} \\
+C w^{2 \theta}[1+|v|]^{\gamma+2}\left|\left[\mathbf{I}-\mathbf{P}_{v}\right] \partial_{j} \partial_{\beta-\beta_{1}}^{\alpha-\alpha_{1}} g_{2}\right|\left|\left[\mathbf{I}-\mathbf{P}_{v}\right] \partial_{i} \partial_{\beta}^{\alpha} g_{3}\right| .
\end{gathered}
$$

Next, we estimate the third term in (3.22). Using the region, we have

$$
\frac{1}{2}|v| \leq|v|-\left|v^{\prime}\right| \leq|\bar{v}| \leq|v|+\left|v^{\prime}\right| \leq \frac{3}{2}|v| \text {. }
$$

Thus we have

$$
\left|\partial_{k l} \phi^{i j}(\bar{v})\right| \leq C[1+|v|]^{\gamma}
$$

and

$$
\left|w^{2 \theta} \partial_{k l} \phi^{i j}(\bar{v}) \partial_{j} \partial_{\beta-\beta_{1}}^{\alpha-\alpha_{1}} g_{2}(v) \partial_{i} \partial_{\beta}^{\alpha} g_{3}(v)\right| \leq C w^{2 \theta}[1+|v|]^{\gamma}\left|\partial_{j} \partial_{\beta-\beta_{1}}^{\alpha-\alpha_{1}} g_{2} \partial_{i} \partial_{\beta}^{\alpha} g_{3}\right| .
$$


Combining (3.22), (3.24), (3.25) and (3.27), we have

$$
\begin{aligned}
& \int\left|\sum_{i j} w^{2 \theta} \partial_{k} \phi^{i j}\left(v-v^{\prime}\right) \partial_{j} \partial_{\beta-\beta_{1}}^{\alpha-\alpha_{1}} g_{2}(v) \partial_{i} \partial_{\beta}^{\alpha} g_{3}(v)\right| d v \\
\leq & C\left[1+\left|v^{\prime}\right|\right]^{2} \int\left|\sum_{i j} w^{2 \theta} \phi^{i j}(v) \partial_{j} \partial_{\beta-\beta_{1}}^{\alpha-\alpha_{1}} g_{2}(v) \partial_{i} \partial_{\beta}^{\alpha} g_{3}(v)\right| d v \\
& +C\left[1+\left|v^{\prime}\right|\right]^{2} \int\left|\sum_{i j} w^{2 \theta} \partial_{k} \phi^{i j}(v) \partial_{j} \partial_{\beta-\beta_{1}}^{\alpha-\alpha_{1}} g_{2}(v) \partial_{i} \partial_{\beta}^{\alpha} g_{3}(v)\right| d v \\
& +C\left[1+\left|v^{\prime}\right|\right]^{2} \int\left|\sum_{i j} w^{2 \theta} \partial_{k l} \phi^{i j}(\bar{v}) \partial_{j} \partial_{\beta-\beta_{1}}^{\alpha-\alpha_{1}} g_{2}(v) \partial_{i} \partial_{\beta}^{\alpha} g_{3}(v)\right| d v \\
\leq & C\left[1+\left|v^{\prime}\right|\right]^{2}\left|w^{\theta} \partial_{\beta-\beta_{1}}^{\alpha-\alpha_{1}} g_{2}\right|_{\sigma}\left|w^{\theta} \partial_{\beta}^{\alpha} g_{3}\right|_{\sigma},
\end{aligned}
$$

where we have used (3.5). Therefore, (3.8) over $\left\{2\left|v^{\prime}\right| \leq|v|,|v| \geq 1\right\}$ is bounded by

$$
\begin{aligned}
& C w^{2 \theta} \int\left[1+\left|v^{\prime}\right|\right]^{2}\left|\partial_{\beta_{1}}\left[\mu^{1 / 2}\left(v^{\prime}\right) \partial^{\alpha_{1}} g_{1}\left(v^{\prime}\right)\right]\right| d v^{\prime} \times\left|w^{\theta} \partial_{\beta-\beta_{1}}^{\alpha-\alpha_{1}} g_{2}\right|_{\sigma}\left|w^{\theta} \partial_{\beta}^{\alpha} g_{3}\right|_{\sigma} \\
\leq & C\left|\partial^{\alpha_{1}} a(x)\right| \int\left[1+\left|v^{\prime}\right|\right]^{2}\left|\partial_{\beta_{1}}\left[\mu^{1 / 2}\left(v^{\prime}\right) \mu^{1 / 4}\left(v^{\prime}\right)\right]\right| d v^{\prime} \times\left|w^{\theta} \partial_{\beta-\beta_{1}}^{\alpha-\alpha_{1}} g_{2}\right|_{\sigma}\left|w^{\theta} \partial_{\beta}^{\alpha} g_{3}\right|_{\sigma} \\
\leq & C\left|\partial^{\alpha_{1}} a(x) \| w^{\theta} \partial_{\beta-\beta_{1}}^{\alpha-\alpha_{1}} g_{2}\right|_{\sigma}\left|w^{\theta} \partial_{\beta}^{\alpha} g_{3}\right|_{\sigma},
\end{aligned}
$$

where we have used $g_{1}(x, v)=a(x) \mu^{1 / 4}(v)$.

Now we consider the second term in (3.9). We again expand $\phi^{i j}\left(v-v^{\prime}\right)$ as

$$
\phi^{i j}\left(v-v^{\prime}\right)=\phi^{i j}(v)-\sum_{k} \partial_{k} \phi^{i j}(\bar{v}) v_{k}^{\prime},
$$

with $\bar{v}$ between $v$ and $v-v^{\prime}$. Since $\sum_{j} \phi^{i j}(v) v_{j}=0$, we obtain

$$
\begin{gathered}
\sum_{j} w^{2 \theta} \phi^{i j}(v) \partial_{j} \partial_{\beta-\beta_{1}}^{\alpha-\alpha_{1}} g_{2}(v) \partial_{\beta}^{\alpha} g_{3}(v) \\
=\sum_{j} w^{2 \theta} \phi^{i j}(v)\left[\mathbf{I}-\mathbf{P}_{v}\right] \partial_{j} \partial_{\beta-\beta_{1}}^{\alpha-\alpha_{1}} g_{2}(v) \partial_{\beta}^{\alpha} g_{3}(v) \\
\leq C w^{2 \theta}[1+|v|]^{\gamma+2}\left|\left[\mathbf{I}-\mathbf{P}_{v}\right] \partial_{j} \partial_{\beta-\beta_{1}}^{\alpha-\alpha_{1}} g_{2}(v)\right|\left|\partial_{\beta}^{\alpha} g_{3}(v)\right| \\
\leq C\left|w^{\theta}[1+|v|]^{\frac{\gamma+2}{2}}\left[\mathbf{I}-\mathbf{P}_{v}\right] \partial_{j} \partial_{\beta-\beta_{1}}^{\alpha-\alpha_{1}} g_{2}(v) \| w^{\theta}[1+|v|]^{\frac{\gamma+2}{2}} \partial_{\beta}^{\alpha} g_{3}(v)\right| .
\end{gathered}
$$

From (3.26), $\left|\partial_{k} \phi^{i j}(\bar{v})\right| \leq C[1+|v|]^{\gamma+1}$. Hence, we have that

$$
\begin{gathered}
\left|w^{2 \theta} \partial_{k} \phi^{i j}(\bar{v}) \partial_{j} \partial_{\beta-\beta_{1}}^{\alpha-\alpha_{1}} g_{2}(v) \partial_{\beta}^{\alpha} g_{3}(v)\right| \\
\leq C w^{2 \theta}[1+|v|]^{\gamma+1}\left|\partial_{j} \partial_{\beta-\beta_{1}}^{\alpha-\alpha_{1}} g_{2}(v)\right|\left|\partial_{\beta}^{\alpha} g_{3}(v)\right| \\
\leq C\left|w^{\theta}[1+|v|]^{\frac{\gamma}{2}} \partial_{j} \partial_{\beta-\beta_{1}}^{\alpha-\alpha_{1}} g_{2}(v)\right|\left|w^{\theta}[1+|v|]^{\frac{\gamma+2}{2}} \partial_{\beta}^{\alpha} g_{3}(v)\right| .
\end{gathered}
$$

From (3.28) and (3.29), we thus conclude

$$
\int\left|\sum_{j} w^{2 \theta} \phi^{i j}\left(v-v^{\prime}\right) \partial_{j} \partial_{\beta-\beta_{1}}^{\alpha-\alpha_{1}} g_{2}(v) \partial_{\beta}^{\alpha} g_{3}(v)\right| d v \leq C\left|w^{\theta} \partial_{\beta-\beta_{1}}^{\alpha-\alpha_{1}} g_{2}\right|_{\sigma}\left|w^{\theta} \partial_{\beta}^{\alpha} g_{3}\right|_{\sigma}
$$


We conclude that (3.9) over $\left\{2\left|v^{\prime}\right| \leq|v|,|v| \geq 1\right\}$ can be majorized by

$$
\begin{aligned}
& \iint\left|w^{2 \theta} \phi^{i j}\left(v-v^{\prime}\right) \partial_{\beta_{1}}\left[v_{i}^{\prime} \mu^{1 / 2}\left(v^{\prime}\right) \partial^{\alpha_{1}} g_{1}\left(v^{\prime}\right)\right] \partial_{j} \partial_{\beta-\beta_{1}}^{\alpha-\alpha_{1}} g_{2}(v) \partial_{\beta}^{\alpha} g_{3}(v)\right| d v^{\prime} d v \\
= & \iint\left|w^{2 \theta}\left[\phi^{i j}(v)-\partial_{k} \phi^{i j}(\bar{v}) v_{k}^{\prime}\right] \partial_{\beta_{1}}\left[v_{i}^{\prime} \mu^{1 / 2}\left(v^{\prime}\right) \partial^{\alpha_{1}} g_{1}\left(v^{\prime}\right)\right]\right| \\
\times & \times \partial_{j} \partial_{\beta-\beta_{1}}^{\alpha-\alpha_{1}} g_{2}(v) \partial_{\beta}^{\alpha} g_{3}(v) \mid d v^{\prime} d v \\
\leq & \left.C \int\left[1+\left|v^{\prime}\right|\right]\left|\partial^{\alpha_{1}} a(x)\right| \partial_{\beta_{1}}\left[v_{i}^{\prime} \mu^{3 / 4}\left(v^{\prime}\right)\right]\left|d v^{\prime}\right| w^{\theta} \partial_{\beta-\beta_{1}}^{\alpha-\alpha_{1}} g_{2}\right|_{\sigma}\left|w^{\theta} \partial_{\beta}^{\alpha} g_{3}\right|_{\sigma} \\
\leq & C\left|\partial^{\alpha_{1}} a(x)\right|\left|w^{\theta} \partial_{\beta-\beta_{1}}^{\alpha-\alpha_{1}} g_{2}\right|_{\sigma}\left|w^{\theta} \partial_{\beta}^{\alpha} g_{3}\right|_{\sigma},
\end{aligned}
$$

where we have used the Cauchy-Schwartz inequality and $g_{1}(x, v)=a(x) \mu^{1 / 4}(v)$.

We now consider the third term in (3.10) over $\left\{2\left|v^{\prime}\right| \leq|v|,|v| \geq 1\right\}$. We use integration by parts to split (3.10) into two parts:

$$
\phi^{i j} * \partial_{\beta_{1}}\left[\mu^{1 / 2} \partial_{j} \partial^{\alpha_{1}} g_{1}\right]=\partial_{j} \phi^{i j} * \partial_{\beta_{1}}\left[\mu^{1 / 2} \partial^{\alpha_{1}} g_{1}\right]-\phi^{i j} * \partial_{\beta_{1}}\left[\partial_{j} \mu^{1 / 2} \partial^{\alpha_{1}} g_{1}\right] .
$$

We first decompose

$$
\partial_{i} \partial_{\beta}^{\alpha} g_{3}=\mathbf{P}_{v} \partial_{i} \partial_{\beta}^{\alpha} g_{3}+\left[\mathbf{I}-\mathbf{P}_{v}\right] \partial_{i} \partial_{\beta}^{\alpha} g_{3}
$$

By similar estimates to (3.28) and (3.29), the second part of (3.10) over $\left\{2\left|v^{\prime}\right| \leq|v|,|v| \geq\right.$ $1\}$ can be estimated as

$$
\begin{gathered}
\iint\left|w^{2 \theta} \phi^{i j}\left(v-v^{\prime}\right) \partial_{\beta_{1}}\left[\partial_{j} \mu^{1 / 2}\left(v^{\prime}\right) \partial^{\alpha_{1}} g_{1}\left(v^{\prime}\right)\right] \partial_{\beta-\beta_{1}}^{\alpha-\alpha_{1}} g_{2}(v) \partial_{i} \partial_{\beta}^{\alpha} g_{3}(v)\right| d v^{\prime} d v \\
=\iint\left|w^{2 \theta}\left[\phi^{i j}(v)-\partial_{k} \phi^{i j}(\bar{v}) v_{k}^{\prime}\right] \partial_{\beta_{1}}\left[\partial_{j} \mu^{1 / 2}\left(v^{\prime}\right) \partial^{\alpha_{1}} a(x) \mu^{1 / 4}\left(v^{\prime}\right)\right]\right| \\
\times\left|\partial_{\beta-\beta_{1}}^{\alpha-\alpha_{1}} g_{2}(v) \partial_{i} \partial_{\beta}^{\alpha} g_{3}(v)\right| d v^{\prime} d v \\
\leq C\left|\partial^{\alpha_{1}} a(x)\right|\left|w^{\theta}[1+|v|]^{\frac{\gamma+2}{2}} \partial_{\beta-\beta_{1}}^{\alpha-\alpha_{1}} g_{2}\right|_{2}\left|w^{\theta}[1+|v|]^{\frac{\gamma+2}{2}}\left[\mathbf{I}-\mathbf{P}_{v}\right] \partial_{i} \partial_{\beta}^{\alpha} g_{3}\right|_{2} \\
+\left.C\left|\partial^{\alpha_{1}} a(x)\right|\left|w^{\theta}[1+|v|]^{\frac{\gamma+2}{2}} \partial_{\beta-\beta_{1}}^{\alpha-\alpha_{1}} g_{2}\right|\right|_{2}\left|w^{\theta}[1+|v|]^{\frac{\gamma}{2}} \partial_{i} \partial_{\beta}^{\alpha} g_{3}\right|_{2} .
\end{gathered}
$$

By (3.5), this is bounded by the right-hand side of (3.6).

We now turn to the proof of the first part of (3.10). By (3.30), noticing that our integration region implies

$$
\left|\partial_{j} \phi^{i j}\left(v-v^{\prime}\right)\right| \leq C[1+|v|]^{\gamma+1},
$$

the first part of (3.10) over $\left\{2\left|v^{\prime}\right| \leq|v|,|v| \geq 1\right\}$ can be estimated as

$$
\begin{gathered}
\iint w^{2 \theta}\left|\partial_{j} \phi^{i j}\left(v-v^{\prime}\right)\right|\left|\partial_{\beta_{1}}\left[\partial_{j} \mu^{1 / 2}\left(v^{\prime}\right) \partial^{\alpha_{1}} g_{1}\left(v^{\prime}\right)\right] \partial_{\beta-\beta_{1}}^{\alpha-\alpha_{1}} g_{2}(v) \partial_{i} \partial_{\beta}^{\alpha} g_{3}(v)\right| d v^{\prime} d v \\
\leq C\left|\partial^{\alpha_{1}} a(x)\right| \int w^{2 \theta}[1+|v|]^{\gamma+1}\left|\partial_{\beta-\beta_{1}}^{\alpha-\alpha_{1}} g_{2}(v) \partial_{i} \partial_{\beta}^{\alpha} g_{3}(v)\right| d v \\
\leq C\left|\partial^{\alpha_{1}} a(x)\right|\left|w^{\theta}[1+|v|]^{\frac{\gamma+2}{2}} \partial_{\beta-\beta_{1}}^{\alpha-\alpha_{1}} g_{2}\right|_{2}\left|w^{\theta}[1+|v|]^{\frac{\gamma}{2}} \partial_{i} \partial_{\beta}^{\alpha} g_{3}\right|_{2},
\end{gathered}
$$

where we have used $g_{1}(x, v)=a(x) \mu^{1 / 4}(v)$. By (3.5), this is bounded by the right-hand side of (3.6). We conclude our theorem. 
Corollary 3.2. Let $|\alpha|+|\beta| \leq N$ and $|\beta| \leq \theta$. If $g_{1}(x, v)$ belongs to the null space $N$ of $L$, namely, $g_{1}(x, v)=a(x) \sqrt{\mu}+\sum_{j=1}^{3} b_{j}(x) v_{j} \sqrt{\mu}+c(x)|v|^{2} \sqrt{\mu}$, then

$$
\begin{aligned}
\left|\left\langle w^{2 \theta} \partial_{\beta}^{\alpha} \Gamma\left(g_{1}, g_{2}\right), \partial_{\beta}^{\alpha} g_{3}\right\rangle\right| \leq C \sum\left[\left|\partial^{\alpha_{1}} a(x)\right|\right. & \left.+\left|\partial^{\alpha_{1}} b_{j}(x)\right|+\left|\partial^{\alpha_{1}} c(x)\right|\right] \\
& \times\left|w^{\theta} \partial_{\beta-\beta_{1}}^{\alpha-\alpha_{1}} g_{2}(x)\right|_{\sigma}\left|w^{\theta} \partial_{\beta}^{\alpha} g_{3}(x)\right|_{\sigma} .
\end{aligned}
$$

If $g_{2}(x, v)=a(x) \sqrt{\mu}+\sum_{j=1}^{3} b_{j}(x) v_{j} \sqrt{\mu}+c(x)|v|^{2} \sqrt{\mu}$, then

$$
\begin{aligned}
\left|\left\langle w^{2 \theta} \partial_{\beta}^{\alpha} \Gamma\left(g_{1}, g_{2}\right), \partial_{\beta}^{\alpha} g_{3}\right\rangle\right| \leq C \sum\left[\left|\partial^{\alpha-\alpha_{1}} a(x)\right|\right. & \left.+\left|\partial^{\alpha-\alpha_{1}} b_{j}(x)\right|+\left|\partial^{\alpha-\alpha_{1}} c(x)\right|\right] \\
& \times\left|w^{\theta} \partial_{\beta_{1}}^{\alpha_{1}} g_{1}(x)\right|_{\sigma}\left|w^{\theta} \partial_{\beta}^{\alpha} g_{3}(x)\right|_{\sigma},
\end{aligned}
$$

where the summation is over $j,\left|\alpha_{1}\right|+\left|\beta_{1}\right| \leq N$ and $\beta_{1} \leq \beta$.

Lemma 3.3. Let $0<\left|\alpha_{1}\right|+\left|\alpha_{2}\right|=|\alpha| \leq N$. Let $g_{1}(x, v), g_{2}(x, v)$ and $h(v)$ be smooth functions. We have

$$
\begin{array}{r}
\left|\left\langle\Gamma\left(g_{1}, g_{2}\right), h\right\rangle\right|_{2} \leq\left\{\begin{array}{c}
C \sum_{|\beta| \leq 2}\left|\partial_{\beta} h\right|_{\sigma} \sum_{|\alpha| \leq N}\left\|\partial^{\alpha} g_{1}\right\|\left\|g_{2}\right\|_{\sigma}, \\
C \sum_{|\beta| \leq 2}\left|\partial_{\beta} h\right|_{2} \sum_{|\alpha| \leq N}\left\|\partial^{\alpha} g_{2}\right\|\left\|g_{1}\right\|_{\sigma},
\end{array}\right. \\
\leq\left\{\begin{array}{cc}
C \sum_{|\beta| \leq 2}\left|\partial_{\beta} h\right|_{\sigma} \sum_{|\alpha| \leq N}\left\|\partial^{\alpha} g_{1}\right\|\left\|\partial^{\alpha-\alpha_{1}} g_{2}\right\|_{\sigma}, & \text { if }\left|\alpha_{1}\right| \leq \frac{N}{2} ; \\
C \sum_{|\beta| \leq 2}\left|\partial_{\beta} h\right|_{2} \sum_{|\alpha| \leq N}\left\|\partial^{\alpha} g_{2}\right\|\left\|\partial^{\alpha_{1}} g_{1}\right\|_{\sigma}, & \text { if }\left|\alpha_{1}\right|>\frac{N}{2} .
\end{array}\right.
\end{array}
$$

Moreover,

$$
\left.\left|\left\langle L \partial^{\alpha} g, h\right\rangle_{2} \leq C \sum_{|\beta| \leq 2}\right| \partial_{\beta} h\right|_{\sigma}\left\|\partial^{\alpha} g\right\|_{\sigma}
$$

Proof. We begin with the linear term. By (3.1) and (3.2), we have that

$$
L g=-\partial_{i}\left[\sigma^{i j} \partial_{j} g\right]+\sigma^{i j} v_{i} v_{j} g-\partial_{i}\left[v_{j} \sigma^{i j}\right] g+\mu^{-1 / 2} \partial_{i}\left\{\mu\left[\phi^{i j} *\left\{\mu^{1 / 2}\left[\partial_{j} g+v_{j} g\right]\right\}\right]\right\} .
$$

Using integrations by parts, $\left\langle L \partial^{\alpha} g, h\right\rangle$ is given by

$$
\begin{gathered}
\int\left\{-\partial^{\alpha} g \cdot \partial_{j}\left[\sigma^{i j} \partial_{i} h(v)\right]+\sigma^{i j} v_{i} v_{j} \partial^{\alpha} g \cdot h(v)-\partial_{i}\left[v_{j} \sigma^{i j}\right] \partial^{\alpha} g \cdot h(v)\right\} d v \\
-\int \mu\left[\phi^{i j} *\left\{\mu^{1 / 2}\left[\partial_{j} \partial^{\alpha} g+v_{j} \partial^{\alpha} g\right]\right\}\right] \partial_{i}\left[\mu^{-1 / 2} h(v)\right] d v
\end{gathered}
$$

where we implicitly sum over $i, j \in\{1,2,3\}$. Using the Cauchy-Schwartz inequality and the following inequality

$$
\left|\sigma^{i j}\right|+\left|\partial_{j} \sigma^{i j}\right|+\left|\partial_{i}\left[v_{j} \sigma^{i j}\right]\right| \leq C[1+|v|]^{\gamma+2},
$$


we obtain that

$$
\begin{aligned}
\mid \int\left\{-\partial^{\alpha} g \cdot \partial_{j}\left[\sigma^{i j} \partial_{i} h(v)\right]\right\} & \left.d v\right|_{2} \leq C \sum_{|\beta| \leq 2}\left|[1+|v|]^{\frac{\gamma+2}{2}} \partial_{\beta} h\right|_{2}\left\|[1+|v|]^{\frac{\gamma+2}{2}} \partial^{\alpha} g\right\| \\
& \leq C \sum_{|\beta| \leq 2}\left|\partial_{\beta} h\right|_{\sigma}\left\|\partial^{\alpha} g\right\|_{\sigma} \\
\left|\int\left\{\partial_{i}\left[v_{j} \sigma^{i j}\right] \partial^{\alpha} g \cdot h(v)\right\} d v\right|_{2} \leq C\left|[1+|v|]^{\frac{\gamma+2}{2}} h\right|_{2}\left\|[1+|v|]^{\frac{\gamma+2}{2}} \partial^{\alpha} g\right\| & \leq C \sum_{|\beta| \leq 2}\left|\partial_{\beta} h\right|_{\sigma}\left\|\partial^{\alpha} g\right\|_{\sigma}, \\
\left|\int \sigma^{i j} v_{i} v_{j} \partial^{\alpha} g \cdot h(v) d v\right|_{2} & \leq C\left\{\int \sigma^{i j} v_{i} v_{j} h^{2} d v\right\}^{1 / 2}\left|\left\{\int \sigma^{i j} v_{i} v_{j}\left|\partial^{\alpha} g\right|^{2} d v\right\}^{1 / 2}\right|_{2} \\
& \leq C \sum_{|\beta| \leq 2}\left|\partial_{\beta} h\right|_{\sigma}\left\|\partial^{\alpha} g\right\|_{\sigma},
\end{aligned}
$$

where we have used the definition of the norm $\|\cdot\|_{\sigma}$.

Using the inequality (3.21), we have that the second line of (3.36) is bounded by

$$
\begin{array}{rl} 
& C\left\|\partial^{\alpha} g\right\|_{\sigma}\left|\int \mu^{1 / 4}[1+|v|]^{\gamma+2}\left[|h|+\left|\partial_{i} h\right|\right] d v\right|_{2} \\
\leq C & C\left\|\partial^{\alpha} g\right\|_{\sigma} \\
& \left\{\int \mu^{1 / 4}[1+|v|]^{2 \gamma+4} d v\right\}^{1 / 2} \\
& \times\left\{\int \mu^{1 / 4}\left[|h|^{2}+\left|\partial_{i} h\right|^{2}\right] d v\right\}^{1 / 2} \\
\leq & C \sum_{|\beta| \leq 2}\left|\partial_{\beta} h\right|_{\sigma}\left\|\partial^{\alpha} g\right\|_{\sigma} .
\end{array}
$$

This completes the proof of estimate (3.35).

Recalling (3.3), $\Gamma\left(\partial^{\alpha_{1}} g_{1}, \partial^{\alpha-\alpha_{1}} g_{2}\right) h$ takes the form

$$
\begin{aligned}
& \partial_{i}\left[\left\{\phi^{i j} *\left[\mu^{1 / 2} \partial^{\alpha_{1}} g_{1}\right]\right\} \partial_{j} \partial^{\alpha-\alpha_{1}} g_{2}\right] h-\left\{\phi^{i j} *\left[v_{i} \mu^{1 / 2} \partial^{\alpha_{1}} g_{1}\right]\right\} \partial_{j} \partial^{\alpha-\alpha_{1}} g_{2} h \\
& -\partial_{i}\left[\left\{\phi^{i j} *\left[\mu^{1 / 2} \partial_{j} \partial^{\alpha_{1}} g_{1}\right]\right\} \partial^{\alpha-\alpha_{1}} g_{2}\right] h+\left\{\phi^{i j} *\left[v_{i} \mu^{1 / 2} \partial_{j} \partial^{\alpha_{1}} g_{1}\right]\right\} \partial^{\alpha-\alpha_{1}} g_{2} h \\
& \quad \equiv I_{1}+I_{2}+I_{3}+I_{4} .
\end{aligned}
$$

We first consider the term $I_{1}$. We rewrite $I_{1}$ as

$$
\begin{aligned}
I_{1}= & \partial_{i}\left[\left\{\phi^{i j} *\left[\mu^{1 / 2} \partial^{\alpha_{1}} g_{1}\right]\right\} \partial_{j} \partial^{\alpha-\alpha_{1}} g_{2} h\right]-\partial_{j}\left[\left\{\phi^{i j} *\left[\mu^{1 / 2} \partial^{\alpha_{1}} g_{1}\right]\right\} \partial^{\alpha-\alpha_{1}} g_{2} \partial_{i} h\right] \\
& +\left\{\partial_{j} \phi^{i j} *\left[\mu^{1 / 2} \partial^{\alpha_{1}} g_{1}\right]\right\} \partial^{\alpha-\alpha_{1}} g_{2} \partial_{i} h+\left\{\phi^{i j} *\left[\mu^{1 / 2} \partial^{\alpha_{1}} g_{1}\right]\right\} \partial^{\alpha-\alpha_{1}} g_{2} \partial_{i j} h .
\end{aligned}
$$

The Cauchy-Schwartz inequality implies that

$$
\begin{aligned}
\left\{\partial_{j} \phi^{i j} *\left[\mu^{1 / 2} \partial^{\alpha_{1}} g_{1}\right]\right\} & \leq\left\{\left|\partial_{j} \phi^{i j}\right|^{2} * \mu^{1 / 2}\right\}^{1 / 2}\left\{\int \mu^{1 / 2}\left(v^{\prime}\right)\left|\partial^{\alpha_{1}} g_{1}\left(v^{\prime}\right)\right|^{2} d v^{\prime}\right\}^{1 / 2} \\
& \leq C[1+|v|]^{\gamma+1} \min \left\{\left|\partial^{\alpha_{1}} g_{1}\right|_{2},\left|\partial^{\alpha_{1}} g_{1}\right|_{\sigma}\right\}
\end{aligned}
$$


If $\left|\alpha_{1}\right| \leq N / 2$, by $H^{2}\left(\mathbf{R}^{3}\right) \subset L^{\infty}\left(\mathbf{R}^{3}\right)$, we have that

$$
\sup _{x}\left|\partial^{\alpha_{1}} g(x)\right|_{2}^{2} \leq C \sum_{|\alpha| \leq N}\left|\partial^{\alpha} g(x)\right|_{2}^{2}
$$

If $\left|\alpha_{1}\right| \leq N / 2$, we have, from (3.5) and the Cauchy-Schwartz inequality, that

$$
\begin{aligned}
& \left|\int\left\{\partial_{j} \phi^{i j} *\left[\mu^{1 / 2} \partial^{\alpha_{1}} g_{1}\right]\right\} \partial^{\alpha-\alpha_{1}} g_{2} \partial_{i} h d v\right|_{2} \\
\leq & \left.\left.C|| \partial^{\alpha_{1}} g_{1}\right|_{2} \int[1+|v|]^{\gamma+2}\left|\partial^{\alpha-\alpha_{1}} g_{2}(x, v) \partial_{i} h(v)\right| d v\right|_{2} \\
\leq & \left.\left.C|| \partial^{\alpha_{1}} g_{1}\right|_{2}\left|\partial^{\alpha-\alpha_{1}} g_{2}(x)\right|_{\sigma}\left|\partial_{i} h\right|_{\sigma}\right|_{2} \\
\leq & C \sum_{|\beta| \leq 2}\left|\partial_{\beta} h\right|_{\sigma} \sum_{|\alpha| \leq N}\left\|\partial^{\alpha} g_{1}\right\|\left\|\partial^{\alpha-\alpha_{1}} g_{2}\right\|_{\sigma} .
\end{aligned}
$$

If $\left|\alpha-\alpha_{1}\right| \leq N / 2$, we also have, from $\gamma+1 \leq-1$, that

$$
\begin{aligned}
& \left|\int\left\{\partial_{j} \phi^{i j} *\left[\mu^{1 / 2} \partial^{\alpha_{1}} g_{1}\right]\right\} \partial^{\alpha-\alpha_{1}} g_{2} \partial_{i} h d v\right|_{2} \\
\leq & \left.\left.C|| \partial^{\alpha_{1}} g_{1}(x)\right|_{\sigma} \int[1+|v|]^{\gamma+1}\left|\partial^{\alpha-\alpha_{1}} g_{2}(x, v) \partial_{i} h(v)\right| d v\right|_{2} \\
\leq & \left.\left.C|| \partial^{\alpha_{1}} g_{1}(x)\right|_{\sigma}\left|\partial^{\alpha-\alpha_{1}} g_{2}(x)\right|_{2}\left|\partial_{i} h\right|_{2}\right|_{2} \\
\leq & C \sum_{|\beta| \leq 2}\left|\partial_{\beta} h\right|_{2} \sum_{|\alpha| \leq N}\left\|\partial^{\alpha} g_{2}\right\|\left\|\partial^{\alpha_{1}} g_{1}\right\|_{\sigma} .
\end{aligned}
$$

The fourth term of $I_{1}$ has the same upper bound by the same arguments as above. This completes the proof of (3.34) about the term $I_{1}$.

For the term $I_{2}$, we split $I_{2}$ as

$$
\begin{aligned}
I_{2}=-\partial_{j}\left[\phi^{i j} *\left[v_{i} \mu^{1 / 2} \partial^{\alpha_{1}} g_{1}\right] \partial_{j} \partial^{\alpha-\alpha_{1}} g_{2} h\right] & +\left\{\partial_{j} \phi^{i j} *\left[v_{i} \mu^{1 / 2} \partial^{\alpha_{1}} g_{1}\right]\right\} \partial^{\alpha-\alpha_{1}} g_{2} h \\
& +\left\{\phi^{i j} *\left[v_{i} \mu^{1 / 2} \partial^{\alpha_{1}} g_{1}\right]\right\} \partial^{\alpha-\alpha_{1}} g_{2} \partial_{j} h .
\end{aligned}
$$

By (3.16) and similar arguments as we used for $I_{1}$, we can obtain the estimates of $I_{2}$.

Similarly, we can rewrite $I_{3}$ as

$$
\begin{aligned}
I_{3}=-\partial_{i}\left[\left\{\phi^{i j} *\left[\mu^{1 / 2} \partial_{j} \partial^{\alpha_{1}} g_{1}\right]\right\} \partial^{\alpha-\alpha_{1}} g_{2} h\right] & +\left\{\phi^{i j} *\left[\mu^{1 / 2} \partial_{j} \partial^{\alpha_{1}} g_{1}\right]\right\} \partial^{\alpha-\alpha_{1}} g_{2} \partial_{i} h \\
=-\partial_{i}\left[\left\{\phi^{i j} *\left[\mu^{1 / 2} \partial_{j} \partial^{\alpha_{1}} g_{1}\right]\right\} \partial^{\alpha-\alpha_{1}} g_{2} h\right]+ & \left\{\partial_{j} \phi^{i j} *\left[\mu^{1 / 2} \partial^{\alpha_{1}} g_{1}\right]\right\} \partial^{\alpha-\alpha_{1}} g_{2} \partial_{i} h \\
& -\left\{\phi^{i j} *\left[\partial_{j} \mu^{1 / 2} \partial^{\alpha_{1}} g_{1}\right]\right\} \partial^{\alpha-\alpha_{1}} g_{2} \partial_{i} h .
\end{aligned}
$$

Applying similar estimates as we used for $I_{1}$, we also can obtain (3.34) about the term $I_{3}$. We now split $I_{4}$ as follows:

$$
\left\{\partial_{j} \phi^{i j} *\left[v_{i} \mu^{1 / 2} \partial^{\alpha_{1}} g_{1}\right]\right\} \partial^{\alpha-\alpha_{1}} g_{2} h-\left\{\phi^{i j} *\left[\partial_{j}\left[v_{i} \mu^{1 / 2}\right] \partial^{\alpha_{1}} g_{1}\right]\right\} \partial^{\alpha-\alpha_{1}} g_{2} h \text {. }
$$

Similar arguments as the last two terms of $I_{3}$ imply that (3.34) about the term $I_{4}$ holds. This completes the proof of (3.34). The similar argument as we used for (3.34) implies that (3.33) holds. 
In the following, we recall the basic estimates in 12 .

Lemma 3.4. Let $|\beta|+|\alpha| \leq N$ and $\theta \geq 0$. For any $\eta>0$, there exists $C_{\eta}>0$ such that

$$
\begin{gathered}
\left\langle w^{2 \theta} \partial_{\beta}[L g], \partial_{\beta} g\right\rangle \geq\left|w^{\theta} \partial_{\beta} g\right|_{\sigma}^{2}-\eta \sum_{\left|\beta_{1}\right| \leq|\beta|}\left|w^{\theta} \partial_{\beta_{1}} g\right|_{\sigma}^{2}-C_{\eta}|g|_{\sigma}^{2} \quad(|\beta|>0), \\
\left|\left\langle w^{2 \theta} \partial_{\beta}^{\alpha} \Gamma(f, g), \partial_{\beta}^{\alpha} h\right\rangle\right| \\
\leq C \sum\left[\left|\partial_{\bar{\beta}}^{\alpha_{1}} f\right|_{2, \theta}\left|\partial_{\beta-\beta_{1}}^{\alpha-\alpha_{1}} g\right|_{\sigma, \theta}+\left|\partial_{\bar{\beta}}^{\alpha_{1}} f\right|_{\sigma, \theta}\left|\partial_{\beta-\beta_{1}}^{\alpha-\alpha_{1}} g\right|_{2, \theta}\right]\left|\partial_{\beta}^{\alpha} h\right|_{\sigma, \theta},
\end{gathered}
$$

where the summation is over $\left|\alpha_{1}\right|+\left|\beta_{1}\right| \leq N, \alpha_{1} \leq \alpha, \bar{\beta} \leq \beta_{1} \leq \beta$.

4. Positivity of $L$. In this section, we shall establish the positivity of the linearized operator $L$ for any classical solution $f(t, x, v)$ to $(1.3)$, which plays an important role in obtaining the global existence result of (1.3).

Lemma 4.1. It holds that $\partial^{\alpha} \mathbf{P} f=\mathbf{P} \partial^{\alpha} f$. Moreover, there exists $C>1$ such that

$$
\frac{1}{C}\left\|w^{|\beta|} \partial_{\beta}^{\alpha} \mathbf{P} f\right\|_{w}^{2} \leq\left\|\partial^{\alpha} a\right\|^{2}+\left\|\partial^{\alpha} b\right\|^{2}+\left\|\partial^{\alpha} c\right\|^{2} \leq C\left\|w^{|\beta|} \partial_{\beta}^{\alpha} \mathbf{P} f\right\|_{w}^{2}
$$

for any smooth function $f(t, x, v)$ and any multi-indices $\beta$.

Proof. A direct computation implies that $\partial^{\alpha} \mathbf{P} f=\mathbf{P} \partial^{\alpha} f$. We plug the expression $w^{|\beta|} \partial_{\beta}^{\alpha} \mathbf{P} f=w^{|\beta|} \partial^{\alpha} a(t, x) \partial_{\beta}\left[\mu^{1 / 2}\right]+w^{|\beta|} \partial^{\alpha} b(t, x) \cdot \partial_{\beta}\left[v \mu^{1 / 2}\right]+w^{|\beta|} \partial^{\alpha} c(t, x) \partial_{\beta}\left[|v|^{2} \mu^{1 / 2}\right]$ into the norms $\|\cdot\|_{\nu}$ and $\|\cdot\|_{\sigma}$. Using $\nu(v) \leq C[1+|v|]^{\gamma},\left|\sigma^{i j}\right| \leq C[1+|v|]^{\gamma+2}$ and the exponential decay of $\mu$, we can obtain the first half of (4.1) by a direct computation. For the second half of (4.1), since $a, b$ and $c$ are the coefficients of a basis to the finite dimensional space $N,\left|\partial^{\alpha} a\right|^{2}+\left|\partial^{\alpha} b\right|^{2}+\left|\partial^{\alpha} c\right|^{2}$ is bounded by $C \int_{\mathbf{R}^{3}} w^{2|\beta|} \nu(v)\left|\partial_{\beta}^{\alpha} \mathbf{P} f\right|^{2} d v$, $C \sum_{i, j=1}^{3} \int_{\mathbf{R}^{3}} w^{2|\beta|} \sigma^{i j} \partial_{i} \partial_{\beta}^{\alpha} \mathbf{P} f \partial_{j} \partial_{\beta}^{\alpha} \mathbf{P} f d v$ and $C \sum_{i, j=1}^{3} \int_{\mathbf{R}^{3}} w^{2|\beta|} \sigma^{i j} v_{i} v_{j}\left|\partial_{\beta}^{\alpha} \mathbf{P} f\right|^{2} d v$ for any $(t, x)$. We then deduce (4.1) by a further integration over $x$.

We know that $\mathbf{P}$ is a projection from $L^{2}\left(\mathbf{R}^{3}\right)$ to the null space $N$ of the linearized operator $L$. Thus for any fixed $(t, x)$, we can decompose any function $f(t, x, v)$ uniquely as

$$
f(t, x, v)=\{\mathbf{P} f\}(t, x, v)+\{\mathbf{I}-\mathbf{P}\} f(t, x, v),
$$

where $\mathbf{P} f$ is called the hydrodynamic part, and $\{\mathbf{I}-\mathbf{P}\} f$ is called the microscopic part 14, 21, 22. We plug $f=\{\mathbf{P} f\}+\{\mathbf{I}-\mathbf{P}\} f$ into equation (1.3). By separating its linear and nonlinear parts, and using $L\{\mathbf{P} f\}=0$, we can express the hydrodynamic part through the microscopic part $\{\mathbf{I}-\mathbf{P}\} f$ :

$$
\left[\partial_{t}+v \cdot \nabla_{x}\right] \mathbf{P} f=l(\{\mathbf{I}-\mathbf{P}\} f)+h(f),
$$

where $l(\{\mathbf{I}-\mathbf{P}\} f)=-\left[\partial_{t}+v \cdot \nabla_{x}+L\right]\{\mathbf{I}-\mathbf{P}\} f$, and $h(f)=\Gamma(f, f)$.

By further expanding $\mathbf{P} f$ as a linear combination of the basis in (1.4),

$$
\left[a(t, x)+\sum_{j=1}^{3} b_{j}(t, x) v_{j}+c(t, x)|v|^{2}\right] \sqrt{\mu},
$$


we can derive the macroscopic equations for $\mathbf{P} f$ 's coefficients $a, b$ and $c$. In fact, the left-hand side of (4.2) now becomes

$$
\sum_{i}\left[v_{i} \partial^{i} c|v|^{2}+\left[\partial^{0} c+\partial^{i} b_{i}\right] v_{i}^{2}+\sum_{j>i}\left[\partial^{i} b_{j}+\partial^{j} b_{i}\right] v_{i} v_{j}+\left[\partial^{0} b_{i}+\partial^{i} a\right] v_{i}+\partial^{0} a\right] \sqrt{\mu},
$$

where $\partial^{0}=\partial_{t}, \partial^{j}=\partial_{x_{j}}$ and $\partial^{i}=\partial_{x_{i}}$. For fixed $(t, x)$, this is an expansion to the left-hand side of (4.2) with respect to the basis of $(1 \leq i \neq j \leq 3)$ :

$$
\sqrt{\mu}, \quad v_{i} \sqrt{\mu}, \quad v_{i}^{2} \sqrt{\mu}, \quad v_{i} v_{j} \sqrt{\mu}, \quad|v|^{2} v_{i} \sqrt{\mu} .
$$

We denote an orthogonal basis for this 13-dimensional space by $\epsilon_{j}, 1 \leq j \leq 13$ as in 9]. We expand the right-hand side of (4.2) with respect to the same basis, and compare with their coefficients on both sides. Then we have

(1) $\nabla_{x} c=l_{c}+h_{c}, \quad(2) \partial^{0} c+\partial^{i} b_{i}=l_{i}+h_{i}$,

$$
\text { (3) } \partial^{i} b_{j}+\partial^{j} b_{i}=l_{i j}+h_{i j}, i \neq j \quad \text { (4) } \partial^{0} b_{i}+\partial^{i} a=l_{b i}+h_{b i}, \quad \text { (5) } \partial^{0} a=l_{a}+h_{a} \text {. }
$$

Here $l_{c}(t, x), l_{i}(t, x), l_{i j}(t, x), l_{b i}(t, x)$ and $l_{a}(t, x)$ are the corresponding coefficients of such an expansion to the linear term $-\left[\partial_{t}+v \cdot \nabla_{x}+L\right]\{\mathbf{I}-\mathbf{P}\} f$, while $h_{c}(t, x), h_{i}(t, x)$, $h_{i j}(t, x), h_{b i}(t, x)$ and $h_{a}(t, x)$ are the corresponding coefficients of the same expansion of the higher-order term $\Gamma(f, f)$.

Now we estimate the $L^{2}$ norm of $l(\{\mathbf{I}-\mathbf{P}\} f)$ through the macroscopic equations (1-5).

LEmma 4.2. Let $\alpha=\left[\alpha_{0}, \alpha_{1}, \alpha_{1}, \alpha_{2}\right]$; then for any $1 \leq i, j \leq 3$,

$$
\sum_{|\alpha| \leq N-1}\left[\left\|\partial^{\alpha} l_{c}\right\|+\left\|\partial^{\alpha} l_{i}\right\|+\left\|\partial^{\alpha} l_{i j}\right\|+\left\|\partial^{\alpha} l_{b i}\right\|+\left\|\partial^{\alpha} l_{a}\right\|\right] \leq C \sum_{|\alpha| \leq N}\left\|\{\mathbf{I}-\mathbf{P}\} \partial^{\alpha} f\right\|_{w}
$$

Proof. We first normalize the basis $\left\{\epsilon_{j}\right\}(1 \leq j \leq 13)$. Let

$$
\left[\mu^{1 / 2}, v_{i} \mu^{1 / 2}, v_{i}^{2} \mu^{1 / 2}, v_{i} v_{j} \mu^{1 / 2},|v|^{2} v_{i} \mu^{1 / 2}\right] A_{13 \times 13}=\left[\epsilon_{j}^{*}\right],
$$

with $\operatorname{det} A \neq 0[9]$. Then for any fixed $(t, x), l_{c}(t, x), l_{i}(t, x), l_{i j}(t, x), l_{b i}(t, x)$ and $l_{a}(t, x)$ take the form

$$
\sum_{i, n=1}^{13} \lambda^{i j} \lambda^{i n} \int_{\mathbf{R}^{3}} l(\{\mathbf{I}-\mathbf{P}\} f) \epsilon_{n}(v) d v
$$

where $\lambda^{i j}$ and $\lambda^{i n}$ are the entries of the matrix $A$.

The same is true after we take $\partial^{\alpha}$. Let $|\alpha| \leq N-1$. Notice that

$$
\int_{\mathbf{R}^{3}} \partial^{\alpha} l(\{\mathbf{I}-\mathbf{P}\} f) \epsilon_{n}(v) d v=-\int_{\mathbf{R}^{3}}\left\{\partial_{t}+v \cdot \nabla_{x}+L\right\}\{\mathbf{I}-\mathbf{P}\} \partial^{\alpha} f(v) \cdot \epsilon_{n}(v) d v .
$$

We now estimate the first two terms:

$$
\begin{aligned}
& \left\|\left\langle\left\{\partial_{t}+v \cdot \nabla_{x}\right\}\{\mathbf{I}-\mathbf{P}\} \partial^{\alpha} f(v), \epsilon_{n}(v)\right\rangle\right\|^{2} \\
\leq & 2 \int_{\mathbf{R}^{3}}\left|\epsilon_{n}(v)\right| d v \int_{\mathbf{R}^{3} \times \mathbf{R}^{3}}\left|\epsilon_{n}(v)\right|\left(\left|\{\mathbf{I}-\mathbf{P}\} \partial^{0} \partial^{\alpha} f\right|^{2}+|v|^{2}\left|\{\mathbf{I}-\mathbf{P}\} \nabla_{x} \partial^{\alpha} f\right|^{2}\right) d x d v \\
\leq & C\left\{\left\|\{\mathbf{I}-\mathbf{P}\} \partial^{0} \partial^{\alpha} f\right\|_{w}+\left\|\{\mathbf{I}-\mathbf{P}\} \nabla_{x} \partial^{\alpha} f\right\|_{w}\right\}^{2},
\end{aligned}
$$

where $\partial^{0}=\partial_{t}$, and we have used (3.5) and the exponential decay of $\epsilon_{n}(v)$. 
By (2.11) in Lemma 2.4 and (3.35) in Lemma 3.3, we have that

$$
\left\|\left\langle L\{\mathbf{I}-\mathbf{P}\} \partial^{\alpha} f, \epsilon_{n}\right\rangle\right\| \leq C\left\|\{\mathbf{I}-\mathbf{P}\} \partial^{\alpha} f\right\|_{w} .
$$

We next estimate the coefficients of the higher-order term $h(f)$. For convenience, we define the temporary energy norm:

$$
[[f]]_{w}^{2}=\|\{\mathbf{I}-\mathbf{P}\} f(t)\|_{w}^{2}+\sum_{|\alpha| \leq N, \alpha \neq 0}\left\|\partial^{\alpha} f(t)\right\|_{w}^{2} .
$$

Noticing that the weaker dissipation norm $[[f]]_{w}^{2}$ without the $L^{2}$ norms of $a, b$ and $c$, we carefully estimate coefficients of the higher-order terms in macroscopic equations.

Lemma 4.3. Let $\alpha=\left[\alpha_{0}, \alpha_{1}, \alpha_{1}, \alpha_{2}\right]$; then for any $1 \leq i, j \leq 3$,

$$
\sum_{|\alpha| \leq N}\left[\left\|\partial^{\alpha} h_{c}\right\|+\left\|\partial^{\alpha} h_{i}\right\|+\left\|\partial^{\alpha} h_{i j}\right\|+\left\|\partial^{\alpha} h_{b i}\right\|+\left\|\partial^{\alpha} h_{a}\right\|\right] \leq C\left|\left\|f|\||_{|\beta|} \cdot[[f]]_{w} .\right.\right.
$$

Proof. Let $|\alpha| \leq N$. Notice that $\partial^{\alpha} h_{c}, \partial^{\alpha} h_{i}, \partial^{\alpha} h_{i j}, \partial^{\alpha} h_{b i}$ and $\partial^{\alpha} h_{a}$ are again of the form

$$
\sum_{i, n=1}^{13} \lambda^{i j} \lambda^{i n} \int_{\mathbf{R}^{3}} \partial^{\alpha} \Gamma(f, f) \cdot \epsilon_{n}(v) d v
$$

where $\lambda^{i j}$ and $\lambda^{i n}$ are the entries of the matrix $A$. It again suffices to estimate $\left\langle\partial^{\alpha} \Gamma(f, f), \epsilon_{n}\right\rangle$. If $0<|\alpha| \leq N$, then we are able to apply (2.8) for the Boltzmann collision operator to get

$$
\begin{aligned}
\left\|\left\langle\Gamma\left(\partial^{\alpha_{1}} f, \partial^{\alpha-\alpha_{1}} f\right), \epsilon_{n}\right\rangle\right\| & \leq C\left[\sum_{\left|\alpha_{i}\right|+\left|\beta_{i}\right| \leq N}\left\|w^{\left|\beta_{i}\right|} \partial_{\beta_{i}}^{\alpha_{i}} f\right\|\right]\left\|\partial^{\alpha-\alpha_{1}} f\right\|_{\nu} \\
& \leq\left|\|f \mid\| \|_{|\beta|} \cdot[[f]]_{\nu},\right.
\end{aligned}
$$

and we are able to apply (3.34) for the Landau collision operator to get

$$
\begin{aligned}
\left\|\left\langle\Gamma\left(\partial^{\alpha_{1}} f, \partial^{\alpha-\alpha_{1}} f\right), \epsilon_{n}\right\rangle\right\| & \leq C\left[\sum_{\left|\alpha_{1}\right| \leq N}\left\|\partial^{\alpha} f\right\|\right]\left\|\partial^{\alpha-\alpha_{1}} f\right\|_{\sigma} \\
& \leq\||f|\|_{|\beta|} \cdot[[f]]_{\sigma},
\end{aligned}
$$

where, without loss of generality, we have assumed that $\left|\alpha-\alpha_{1}\right|>0$.

In the following, we consider the case $\alpha=0$. We split $f=\mathbf{P} f+\{\mathbf{I}-\mathbf{P}\} f$ so that $\left\|\left\langle\Gamma(f, f), \epsilon_{n}\right\rangle\right\|$ is decomposed into

$$
\begin{aligned}
\left\|\left\langle\Gamma(\mathbf{P} f,\{\mathbf{I}-\mathbf{P}\} f), \epsilon_{n}\right\rangle\right\|+\left\|\left\langle\Gamma(\{\mathbf{I}-\mathbf{P}\} f, \mathbf{P} f), \epsilon_{n}\right\rangle\right\| \\
+\left\|\left\langle\Gamma(\{\mathbf{I}-\mathbf{P}\} f,\{\mathbf{I}-\mathbf{P}\} f), \epsilon_{n}\right\rangle\right\|+\left\|\left\langle\Gamma(\mathbf{P} f, \mathbf{P} f), \epsilon_{n}\right\rangle\right\| .
\end{aligned}
$$

For the Boltzmann collision operator, by applying (2.7) in Lemma 2.3, we easily obtain that the first three terms are bounded by

$$
C\left[\sum_{\left|\alpha_{i}\right|+\left|\beta_{i}\right| \leq N}\left\|w^{\left|\beta_{i}\right|} \partial_{\beta_{i}}^{\alpha_{i}} f\right\|\right]\|\{\mathbf{I}-\mathbf{P}\} f\|_{\nu} \leq C\left|\|f \mid\| \|_{|\beta|} \cdot[[f]]_{\nu} .\right.
$$


For the Landau collision operator, by applying (3.33) in Lemma 3.3, we easily obtain that the first three terms are bounded by

$$
C\left[\sum_{|\alpha| \leq N}\left\|\partial^{\alpha} f\right\|\right]\|\{\mathbf{I}-\mathbf{P}\} f\|_{\sigma} \leq C\left|\|f \mid\| \|_{|\beta|} \cdot[[f]]_{\sigma} .\right.
$$

On the other hand, we plug $\mathbf{P} f=\left[a(t, x)+b(t, x) \cdot v+c(t, x)|v|^{2}\right] \sqrt{\mu}$ into the last term to get

$$
\left\|\left\langle\Gamma(\mathbf{P} f, \mathbf{P} f), \epsilon_{n}\right\rangle\right\| \leq C\left\|a^{2}+|b|^{2}+c^{2}\right\| .
$$

For the estimate of the terms $a^{2},|b|^{2}$ and $c^{2}$, we shall use the similar method as used in [16]. By the generalized Hölder inequality, we obtain

$$
\begin{aligned}
\| a^{2}+|b|^{2}+ & c^{2} \| \leq C\left\{\|a(t, \cdot)\|_{L^{6}}+\|b(t, \cdot)\|_{L^{6}}+\|c(t, \cdot)\|_{L^{6}}\right\} \\
& \times\left\{\|a(t, \cdot)\|_{L^{3}}+\|b(t, \cdot)\|_{L^{3}}+\|c(t, \cdot)\|_{L^{3}}\right\} .
\end{aligned}
$$

This first factor is bounded by Sobolev's inequality in $\mathbf{R}^{3}$ and Lemma 4.1 as

$$
\left[\left\|\nabla_{x} a(t, \cdot)\right\|+\left\|\nabla_{x} b(t, \cdot)\right\|+\left\|\nabla_{x} c(t, \cdot)\right\|\right] \leq C[[f]]_{w},
$$

while the second factor is bounded by an interpolation as

$$
\left[\|a(t, \cdot)\|_{H^{1}}+\|b(t, \cdot)\|_{H^{1}}+\|c(t, \cdot)\|_{H^{1}}\right] \leq C\left|\|f \mid\|_{|\beta|} \cdot\right.
$$

Thus, we have the following $L^{2}\left(\mathbf{R}^{3}\right)$ estimate for $\Gamma(f, f)$ :

$$
\sum_{|\alpha| \leq N}\left[\left\|\partial^{\alpha} h_{c}\right\|+\left\|\partial^{\alpha} h_{i}\right\|+\left\|\partial^{\alpha} h_{i j}\right\|+\left\|\partial^{\alpha} h_{a i}\right\|+\left\|\partial^{\alpha} h_{a}\right\|\right] \leq C\left|\left\|f|\||_{|\beta|} \cdot[[f]]_{w} .\right.\right.
$$

We thus conclude the proof of Lemma 4.3.

Theorem 4.4. Let $f(t, x, v)$ be the unique classical solution to (1.3). There exist positive constants $M_{0}, \delta_{0}=\delta_{0}\left(M_{0}\right), C_{1}$ and $C_{2}$ such that if

$$
\sum_{|\alpha|+|\beta| \leq N}\left\|w^{|\beta|} \partial_{\beta}^{\alpha} f(t)\right\|^{2} \leq M_{0} / 2,
$$

then

$$
\begin{aligned}
\sum_{0<|\alpha| \leq N}\left(L \partial^{\alpha} f(s), \partial^{\alpha} f(s)\right) \geq \delta_{0} & \sum_{0<|\alpha| \leq N}\left\|\partial^{\alpha} f(s)\right\|_{w}^{2} \\
& \quad-C_{1} \frac{d}{d t} \int_{\mathbf{R}^{3}} \nabla \cdot b c d x-C_{2}\|\{\mathbf{I}-\mathbf{P}\} f\|_{w} .
\end{aligned}
$$

Proof. Since $\left(L \partial^{\alpha} f(s), \partial^{\alpha} f(s)\right) \geq \delta\left\|\{\mathbf{I}-\mathbf{P}\} \partial^{\alpha} f\right\|_{w}^{2}$, it thus suffices to show that if (4.6) is valid for some small $M_{0}>0$, then there is a constant $C>0$ such that

$$
\begin{gathered}
\sum_{0<|\alpha| \leq N}\left\|\mathbf{P} \partial^{\alpha} f\right\|_{w}^{2} \leq C \sum_{0<|\alpha| \leq N}\left\|\{\mathbf{I}-\mathbf{P}\} \partial^{\alpha} f(s)\right\|_{w}^{2}+C_{2}\|\{\mathbf{I}-\mathbf{P}\} f\|_{w}^{2} \\
+C_{1} \frac{d}{d t} \int_{\mathbf{R}^{3}} \nabla \cdot b c d x
\end{gathered}
$$


By Lemma 4.1, equivalently, we therefore need only to establish that

$$
\begin{aligned}
\sum_{0<|\alpha| \leq N}\left\{\left\|\partial^{\alpha} a\right\|+\left\|\partial^{\alpha} b\right\|\right. & \left.+\left\|\partial^{\alpha} c\right\|\right\}^{2} \leq C \sum_{|\alpha| \leq N}\left\|\{\mathbf{I}-\mathbf{P}\} \partial^{\alpha} f(t)\right\|_{w}^{2} \\
& +C\left|\|f \mid\|_{|\beta|}^{2} \cdot[[f]]_{w}^{2}+C_{1} \frac{d}{d t} \int_{\mathbf{R}^{3}} \nabla \cdot b c d x\right.
\end{aligned}
$$

that is,

$$
\begin{aligned}
& \sum_{0<|\alpha| \leq N}\left\{\left\|\partial^{\alpha} a\right\|+\left\|\partial^{\alpha} b\right\|+\left\|\partial^{\alpha} c\right\|\right\}^{2} \\
\leq & C \sum_{|\alpha| \leq N}\left\|\{\mathbf{I}-\mathbf{P}\} \partial^{\alpha} f(t)\right\|_{w}^{2}+C_{1} \frac{d}{d t} \int_{\mathbf{R}^{3}} \nabla \cdot b c d x \\
& +C M_{0}\left\{\sum_{0<|\alpha| \leq N}\left\{\left\|\partial^{\alpha} a\right\|+\left\|\partial^{\alpha} b\right\|+\left\|\partial^{\alpha} c\right\|\right\}+\sum_{|\alpha| \leq N}\left\|\{\mathbf{I}-\mathbf{P}\} \partial^{\alpha} f(t)\right\|_{w}\right\}^{2},
\end{aligned}
$$

which implies (4.7) when $M_{0}$ is sufficiently small. By Lemma 4.2 and Lemma 4.3, similar arguments as those used in [14, 16, imply that (4.7) holds.

5. Global Solutions. In this section we shall derive a refined energy estimate, which is a crucial step in constructing global solutions. We first review the local existence results. For periodic initial data, the local existence result of (1.3) was given in [12, 15]. By a straightforward modification of the arguments there, we have the following local existence result in the whole space.

Lemma 5.1. There exist $M_{0}>0$ and $T^{*}>0$ such that if $T^{*} \leq M_{0} / 2$ and $\mathcal{E}\left(f_{0}\right) \leq M_{0} / 2$, then there is a unique solution $f(t, x, v)$ to $(1.3)$ in $\left[0, T^{*}\right) \times \mathbf{R}^{3} \times \mathbf{R}^{3}$ such that

$$
\frac{1}{2}|||f(t)|\left\|_{|\beta|}^{2}+\sum_{|\alpha|+|\beta| \leq N} \int_{0}^{t}\right\| \partial_{\beta}^{\alpha} f(s) \|_{w,|\beta|}^{2} d s \leq M_{0} .
$$

Moreover, $\left|\|f(t) \mid\|_{|\beta|}^{2}\right.$ is continuous over $\left[0, T^{*}\right)$. If $F_{0}(x, v)=\mu+\sqrt{\mu} f_{0}(x, v) \geq 0$, then $F(t, x, v)=\mu+\sqrt{\mu} f(t, x, v) \geq 0$.

Proof of Theorem 1.1. Taking $\partial^{\alpha}$ on $(1.3)(0<|\alpha| \leq N)$, we obtain

$$
\frac{1}{2} \frac{d}{d t} \sum_{|\alpha| \neq 0}\left\|\partial^{\alpha} f(t)\right\|^{2}+\sum_{|\alpha| \neq 0}\left(L \partial^{\alpha} f, \partial^{\alpha} f\right)=\sum_{|\alpha| \neq 0}\left(\partial^{\alpha} \Gamma(f, f), \partial^{\alpha} f\right) .
$$

The goal is to show that

$$
\left(\partial^{\alpha} \Gamma(f, f), \partial^{\alpha} f\right) \leq C|||f|||_{|\beta|} \cdot|||f| \|_{w,|\beta|}^{2} \cdot
$$

We split $f=\{\mathbf{P} f\}+\{\mathbf{I}-\mathbf{P}\} f$ to further decompose $\left(\partial^{\alpha} \Gamma(f, f), \partial^{\alpha} f\right)$ into

$$
\begin{gathered}
\left(\partial^{\alpha} \Gamma(\mathbf{P} f, \mathbf{P} f), \partial^{\alpha} f\right)+\left(\partial^{\alpha} \Gamma(\{\mathbf{I}-\mathbf{P}\} f, \mathbf{P} f), \partial^{\alpha} f\right) \\
+\left(\partial^{\alpha} \Gamma(\mathbf{P} f,\{\mathbf{I}-\mathbf{P}\} f), \partial^{\alpha} f\right)+\left(\partial^{\alpha} \Gamma(\{\mathbf{I}-\mathbf{P}\} f,\{\mathbf{I}-\mathbf{P}\} f), \partial^{\alpha} f\right) .
\end{gathered}
$$


Now we consider the first part. We plug $\mathbf{P} f=\left[a(t, x)+b(t, x) \cdot v+c(t, x)|v|^{2}\right] \sqrt{\mu}$ into the expression and apply Corollary 2.2, Corollary 3.2 and Lemma 4.1 to get

$$
\begin{aligned}
& \left(\partial^{\alpha} \Gamma(\mathbf{P} f, \mathbf{P} f), \partial^{\alpha} f\right) \\
\leq & C \sum_{\alpha_{1} \leq \alpha} \int_{\mathbf{R}^{3}}\left(\left|\partial^{\alpha_{1}} a\right|+\left|\partial^{\alpha_{1}} b\right|+\left|\partial^{\alpha_{1}} c\right|\right)\left(\left|\partial^{\alpha-\alpha_{1}} a\right|+\left|\partial^{\alpha-\alpha_{1}} b\right|+\left|\partial^{\alpha-\alpha_{1}} c\right|\right)\left|\partial^{\alpha} f\right|_{w} d x \\
\leq & C \sum_{\alpha_{1} \leq \alpha} \sup _{x}\left(\left|\partial^{\alpha_{1}} a\right|+\left|\partial^{\alpha_{1}} b\right|+\left|\partial^{\alpha_{1}} c\right|\right) \int_{\mathbf{R}^{3}}\left(\left|\partial^{\alpha-\alpha_{1}} a\right|+\left|\partial^{\alpha-\alpha_{1}} b\right|+\left|\partial^{\alpha-\alpha_{1}} c\right|\right)\left|\partial^{\alpha} f\right|_{w} d x \\
\leq & C \sum_{\alpha_{1} \leq \alpha|\ell| \leq 2} \sum_{\left.\mid\left\|\partial^{\alpha_{1}+\ell} a\right\|+\left\|\partial^{\alpha_{1}+\ell} b\right\|+\left\|\partial^{\alpha_{1}+\ell} c\right\|\right]} \\
& \quad \times\left[\left\|\partial^{\alpha-\alpha_{1}} a\right\|+\left\|\partial^{\alpha-\alpha_{1}} b\right\|+\left\|\partial^{\alpha-\alpha_{1}} c\right\|\right] \cdot\left\|\partial^{\alpha} f\right\|_{w} \\
\leq & C \sum_{\alpha_{1} \leq \alpha}\left\|\partial^{\alpha_{1}} f\right\|\left\|\partial^{\alpha-\alpha_{1}} f\right\|_{w} \cdot\left\|\partial^{\alpha} f\right\|_{w} \\
\leq & C\left|\|f\|\left\|_{|\beta|} \cdot\right\|\right|\|f\|_{w,|\beta|}^{2},
\end{aligned}
$$

where we have assumed that $\left|\alpha_{1}\right| \leq N / 2$ and used $H^{2}\left(\mathbf{R}^{3}\right) \subset L^{\infty}\left(\mathbf{R}^{3}\right)$.

We apply Corollary 2.2 and Corollary 3.2 to get

$$
\begin{aligned}
& \left(\partial^{\alpha} \Gamma(\{\mathbf{I}-\mathbf{P}\} f, \mathbf{P} f), \partial^{\alpha} f\right)+\left(\partial^{\alpha} \Gamma(\mathbf{P} f,\{\mathbf{I}-\mathbf{P}\} f), \partial^{\alpha} f\right) \\
\leq & \sum_{\alpha_{1} \leq \alpha} \int_{\mathbf{R}^{3}}\left(\left|\partial^{\alpha_{1}} a\right|+\left|\partial^{\alpha_{1}} b\right|+\left|\partial^{\alpha_{1}} c\right|\right)\left|\{\mathbf{I}-\mathbf{P}\} \partial^{\alpha-\alpha_{1}} f\right|_{w}\left|\partial^{\alpha} f\right|_{w} d x \\
& +\sum_{\alpha_{1} \leq \alpha} \int_{\mathbf{R}^{3}}\left(\left|\partial^{\alpha-\alpha_{1}} a\right|+\left|\partial^{\alpha-\alpha_{1}} b\right|+\left|\partial^{\alpha-\alpha_{1}} c\right|\right)\left|\{\mathbf{I}-\mathbf{P}\} \partial^{\alpha_{1}} f\right|_{w}\left|\partial^{\alpha} f\right|_{w} d x .
\end{aligned}
$$

The first summation of the above inequality is bounded by

$$
\begin{gathered}
\sum_{\left|\alpha_{1}\right| \leq|\alpha| / 2} \int_{\mathbf{R}^{3}}\left(\left|\partial^{\alpha_{1}} a\right|+\left|\partial^{\alpha_{1}} b\right|+\left|\partial^{\alpha_{1}} c\right|\right)\left|\{\mathbf{I}-\mathbf{P}\} \partial^{\alpha-\alpha_{1}} f\right|_{w}\left|\partial^{\alpha} f\right|_{w} d x \\
+\sum_{\left|\alpha_{1}\right| \geq|\alpha| / 2} \int_{\mathbf{R}^{3}}\left(\left|\partial^{\alpha_{1}} a\right|+\left|\partial^{\alpha_{1}} b\right|+\left|\partial^{\alpha_{1}} c\right|\right)\left|\{\mathbf{I}-\mathbf{P}\} \partial^{\alpha-\alpha_{1}} f\right|_{w}\left|\partial^{\alpha} f\right|_{w} d x \\
\leq \sum_{\left|\alpha_{1}\right| \leq|\alpha| / 2} \sup _{x}\left(\left|\partial^{\alpha_{1}} a\right|+\left|\partial^{\alpha_{1}} b\right|+\left|\partial^{\alpha_{1}} c\right|\right) \int_{\mathbf{R}^{3}}\left|\{\mathbf{I}-\mathbf{P}\} \partial^{\alpha-\alpha_{1}} f\right|_{w}\left|\partial^{\alpha} f\right|_{w} d x \\
\quad+\sum_{\left|\alpha_{1}\right| \geq|\alpha| / 2} \sup _{x}\left|\{\mathbf{I}-\mathbf{P}\} \partial^{\alpha-\alpha_{1}} f\right|_{w} \int_{\mathbf{R}^{3}}\left(\left|\partial^{\alpha_{1}} a\right|+\left|\partial^{\alpha_{1}} b\right|+\left|\partial^{\alpha_{1}} c\right|\right)\left|\partial^{\alpha} f\right|_{w} d x \\
\leq \sum_{\left|\alpha_{1}\right| \leq|\alpha| / 2}\left[\sum_{|\ell| \leq N}\left\|\partial^{\ell} f\right\|\right]\left\|\{\mathbf{I}-\mathbf{P}\} \partial^{\alpha-\alpha_{1}} f\right\|_{w}\left\|\partial^{\alpha} f\right\|_{w} \\
+\sum_{\left|\alpha_{1}\right| \geq|\alpha| / 2}\left[\sum_{|\ell| \leq N}\left\|\{\mathbf{I}-\mathbf{P}\} \partial^{\ell} f\right\|_{w}\right]\left\|\partial^{\alpha_{1}} f\right\|\left\|\partial^{\alpha} f\right\|_{w} \\
\leq C|||f|\left\|\left|\left\|_{|\beta|} \cdot|||f|\right\|_{w,|\beta|}^{2},\right.\right.
\end{gathered}
$$

where we have used Lemma 4.1 and Sobolev's inequality. 
For the last term of (5.2), we can apply Lemma 2.5 and Lemma 3.4 with $\theta=0$ and it can be bounded by $C\left|\left\|f\left|\left\|_{|\beta|} \cdot|||f|\right\|_{w,|\beta|}^{2}\right.\right.\right.$.

We therefore conclude by Theorem 4.4 that

$$
\begin{gathered}
\frac{d}{d t}\left[\sum_{|\alpha| \neq 0}\left\|\partial^{\alpha} f(t)\right\|^{2}-2 C_{1} \int_{\mathbf{R}^{3}} \nabla \cdot b c d x\right]+\delta_{0} \sum_{|\alpha| \neq 0}\left\|\partial^{\alpha} f(s)\right\|_{w}^{2} \\
\leq C\left|\left\|f\left|\left\|_{|\beta|} \cdot\right\|\right||f|\right\|_{w,|\beta|}^{2}+C\|\{\mathbf{I}-\mathbf{P}\} f\|_{w} .\right.
\end{gathered}
$$

The second step is to treat pure $v$-derivatives of $\{\mathbf{I}-\mathbf{P}\} f$. We first turn (1.3) into a microscopic-type equation:

$$
\left[\partial_{t}+v \cdot \nabla_{x}+L\right]\{\mathbf{I}-\mathbf{P}\} f=-\left[\partial_{t}+v \cdot \nabla_{x}\right] \mathbf{P} f+\Gamma(f, f) .
$$

Taking the pure $\partial_{\beta}(0<|\beta| \leq N)$ derivatives of (5.4), we obtain

$$
\begin{gathered}
{\left[\partial_{t}+v \cdot \nabla_{x}\right] \partial_{\beta}\{\mathbf{I}-\mathbf{P}\} f+\sum_{\left|\beta_{1}\right|=1} C_{\beta}^{\beta_{1}} \partial_{\beta_{1}} v \cdot \nabla_{x} \partial_{\beta-\beta_{1}}\{\mathbf{I}-\mathbf{P}\} f+\partial_{\beta} L\{\mathbf{I}-\mathbf{P}\} f} \\
=\partial_{\beta} \Gamma(f, f)-\left[\partial_{t}+v \cdot \nabla_{x}\right] \partial_{\beta} \mathbf{P} f-\sum_{\left|\beta_{1}\right|=1} C_{\beta}^{\beta_{1}} \partial_{\beta_{1}} v \cdot \nabla_{x} \partial_{\beta-\beta_{1}} \mathbf{P} f
\end{gathered}
$$

By multiplying $w^{2|\beta|} \partial_{\beta}\{\mathbf{I}-\mathbf{P}\} f$ to (5.5) and then integrating over $\mathbf{R}^{3} \times \mathbf{R}^{3}$, we get

$$
\begin{gathered}
\frac{1}{2} \frac{d}{d t}\left\|w^{|\beta|} \partial_{\beta}\{\mathbf{I}-\mathbf{P}\} f\right\|^{2}+\left(w^{2|\beta|} \partial_{\beta} L\{\mathbf{I}-\mathbf{P}\} f, \partial_{\beta}\{\mathbf{I}-\mathbf{P}\} f\right) \\
+\sum_{\left|\beta_{1}\right|=1} C_{\beta}^{\beta_{1}}\left(w^{2|\beta|} \partial_{\beta_{1}} v_{j} \partial^{j} \partial_{\beta-\beta_{1}}\{\mathbf{I}-\mathbf{P}\} f, \partial_{\beta}\{\mathbf{I}-\mathbf{P}\} f\right) \\
+\sum_{\left|\beta_{1}\right|=1} C_{\beta}^{\beta_{1}}\left(w^{2|\beta|} \partial_{\beta_{1}} v_{j} \partial^{j} \partial_{\beta-\beta_{1}} \mathbf{P} f, \partial_{\beta}\{\mathbf{I}-\mathbf{P}\} f\right) \\
=\left(w^{2|\beta|} \partial_{\beta} \Gamma(f, f), \partial_{\beta}\{\mathbf{I}-\mathbf{P}\} f\right)-\left(w^{2|\beta|} \partial_{\beta}\left[\partial_{t}+v \cdot \nabla_{x}\right] \mathbf{P} f, \partial_{\beta}\{\mathbf{I}-\mathbf{P}\} f\right) .
\end{gathered}
$$

We now estimate term by term. Applying (2.12) in Lemma 2.4 and (3.37) in Lemma 3.4 and integrating over $\mathbf{R}^{3}$, we deduce, for any $\eta>0$, that

$$
\begin{aligned}
\left(w^{2|\beta|} \partial_{\beta} L\{\mathbf{I}-\mathbf{P}\} f, \partial_{\beta}\{\mathbf{I}-\mathbf{P}\} f\right) \geq\left\|w^{|\beta|} \partial_{\beta}\{\mathbf{I}-\mathbf{P}\} f\right\|_{w}^{2} & \\
& -\eta \sum_{\left|\beta_{1}\right| \leq|\beta|}\left\|w^{\left|\beta_{1}\right|} \partial_{\beta_{1}}\{\mathbf{I}-\mathbf{P}\} f\right\|_{w}^{2}-C_{\eta}\|\{\mathbf{I}-\mathbf{P}\} f\|_{w}^{2} .
\end{aligned}
$$

We estimate the third term on the left-hand side of (5.6). For any $\eta>0$, we have

$$
\begin{aligned}
& \left(w^{2|\beta|} \partial_{\beta_{1}} v_{j} \partial^{j} \partial_{\beta-\beta_{1}}\{\mathbf{I}-\mathbf{P}\} f, \partial_{\beta}\{\mathbf{I}-\mathbf{P}\} f\right) \\
\leq & \iint w^{2|\beta|}\left|\partial^{j} \partial_{\beta-\beta_{1}}\{\mathbf{I}-\mathbf{P}\} f \partial_{\beta}\{\mathbf{I}-\mathbf{P}\} f\right| d x d v \\
\leq & \left\|w^{1 / 2+|\beta|} \partial_{\beta}\{\mathbf{I}-\mathbf{P}\} f\right\|\left\|w^{1 / 2+\{|\beta|-1\}} \partial_{\beta-\beta_{1}}^{j}\{\mathbf{I}-\mathbf{P}\} f\right\| \\
\leq & \eta\left\|w^{|\beta|} \partial_{\beta}\{\mathbf{I}-\mathbf{P}\} f\right\|_{w}^{2}+C_{\eta}\left\|w^{\left|\beta-\beta_{1}\right|} \partial_{\beta-\beta_{1}}^{j}\{\mathbf{I}-\mathbf{P}\} f\right\|_{w}^{2} .
\end{aligned}
$$

Here $\left|\beta-\beta_{1}\right|=|\beta|-1$. For the Boltzmann equation, we have used the fact that $\|g\|_{\nu}$ is equivalent to $\left\|w^{1 / 2} g\right\|$ for $w=[1+|v|]^{\gamma}$, and for the Landau equation, we have used $\left\|w^{1 / 2} g\right\| \leq C\|g\|_{\sigma}$ by $(3.5)$ for $w=[1+|v|]^{\gamma+2}$. 
By similar arguments as used in the third term and by Lemma 4.1, for any $\eta>0$, the fourth term on the left-hand side of (5.6) is bounded by

$$
\begin{aligned}
& \left(w^{2|\beta|} \partial_{\beta_{1}} v_{j} \partial^{j} \partial_{\beta-\beta_{1}} \mathbf{P} f, \partial_{\beta}\{\mathbf{I}-\mathbf{P}\} f\right) \\
\leq & \eta\left\|w^{|\beta|} \partial_{\beta}\{\mathbf{I}-\mathbf{P}\} f\right\|_{w}^{2}+C_{\eta}\left\|w^{\left|\beta-\beta_{1}\right|} \partial_{\beta-\beta_{1}}^{j} \mathbf{P} f\right\|_{w}^{2} \\
\leq & \eta\left\|w^{|\beta|} \partial_{\beta}\{\mathbf{I}-\mathbf{P}\} f\right\|_{w}^{2}+C_{\eta} \sum_{\left|\alpha_{1}\right|=1}\left\|\partial^{\alpha_{1}} f\right\|_{w}^{2} .
\end{aligned}
$$

By similar arguments as used above, we have that, for any $\eta>0$, the second term on the right-hand side of (5.6) is bounded by

$$
\eta\left\|w^{|\beta|} \partial_{\beta}\{\mathbf{I}-\mathbf{P}\} f\right\|_{w}^{2}+C_{\eta} \sum_{\left|\alpha_{1}\right|=1}\left\|\partial^{\alpha_{1}} f\right\|_{w}^{2} .
$$

Finally, we estimate the nonlinear collision term. Our goal is still to show

$$
\left(w^{2|\beta|} \partial_{\beta} \Gamma(f, f), \partial_{\beta}\{\mathbf{I}-\mathbf{P}\} f\right) \leq C|||f|\left\|_{|\beta|} \cdot\right\||| f \mid \|_{w,|\beta|}^{2} \cdot
$$

We treat this term similarly as (5.2) and first decompose $\left(w^{2|\beta|} \partial_{\beta} \Gamma(f, f), \partial_{\beta}\{\mathbf{I}-\mathbf{P}\} f\right)$ as follows:

$$
\begin{aligned}
& \left(w^{2|\beta|} \partial_{\beta} \Gamma(\mathbf{P} f,\{\mathbf{I}-\mathbf{P}\} f), \partial_{\beta}\{\mathbf{I}-\mathbf{P}\} f\right)+\left(w^{2|\beta|} \partial_{\beta} \Gamma(\{\mathbf{I}-\mathbf{P}\} f, \mathbf{P} f), \partial_{\beta}\{\mathbf{I}-\mathbf{P}\} f\right) \\
+ & \left(w^{2|\beta|} \partial_{\beta} \Gamma(\mathbf{P} f, \mathbf{P} f), \partial_{\beta}\{\mathbf{I}-\mathbf{P}\} f\right)+\left(w^{2|\beta|} \partial_{\beta} \Gamma(\{\mathbf{I}-\mathbf{P}\} f,\{\mathbf{I}-\mathbf{P}\} f), \partial_{\beta}\{\mathbf{I}-\mathbf{P}\} f\right) .
\end{aligned}
$$

For the first term of (5.8), by (2.5) and (3.31) with $\theta=|\beta|$, we have

$$
\begin{gathered}
\leq C \sum_{\left|\beta_{1}\right| \leq|\beta|} \int_{\mathbf{R}^{3}}(|a|+|b|+|c|)\left|w^{\left|\beta_{1}\right|} \partial_{\beta_{1}}\{\mathbf{I}-\mathbf{P}\} f\right|_{w} \mid w^{|\beta|} \partial_{\beta} \Gamma\left(\mathbf{P} f,\left.\{\mathbf{I}-\mathbf{I}\} f\right|_{w} d x\right. \\
\leq C \sup _{x}(|a|+|b|+|c|) \sum_{\left|\beta_{1}\right| \leq|\beta|} \int_{\mathbf{R}^{3}}\left|w^{\left|\beta_{1}\right|} \partial_{\beta_{1}}\{\mathbf{I}-\mathbf{P}\} f\right|_{w}\left|w^{|\beta|} \partial_{\beta}\{\mathbf{I}-\mathbf{P}\} f\right|_{w} d x \\
\leq C \sum_{|\ell| \leq 2}\left\|\partial^{\ell} f\right\| \sum_{\left|\beta_{1}\right| \leq|\beta|}\left\|w^{\left|\beta_{1}\right|} \partial_{\beta_{1}}\{\mathbf{I}-\mathbf{P}\} f\right\|_{w}\left\|w^{|\beta|} \partial_{\beta}\{\mathbf{I}-\mathbf{P}\} f\right\|_{w},
\end{gathered}
$$

where we have used Lemma $4.1, w \leq 1$ and $w^{|\beta|} \leq w^{\left|\beta_{1}\right|}$ for $\beta_{1} \leq \beta$. Obviously, the last line of the above inequality is controlled by $C\left|\left\|\left.f||\right|_{|\beta|}|||f|\right\|_{w,|\beta|}^{2}\right.$.

The second term of (5.8) has the same upper bound as the first term by (2.6), (3.32) with $\theta=|\beta|$ and Lemma 4.1 as well as by Sobolev's inequality.

For the third term $\partial_{\beta} \Gamma(\mathbf{P} f, \mathbf{P} f)$, we apply (2.5) and (3.31) with $\theta=|\beta|$ to get

$$
\begin{aligned}
& \left(w^{2|\beta|} \partial_{\beta} \Gamma(\mathbf{P} f, \mathbf{P} f), \partial_{\beta}\{\mathbf{I}-\mathbf{P}\} f\right) \\
& \leq C \sum_{\left|\beta_{1}\right| \leq|\beta|} \int_{\mathbf{R}^{3}}(|a|+|b|+|c|)\left|w^{\left|\beta_{1}\right|} \partial_{\beta_{1}} \mathbf{P} f\right|_{w}\left|w^{|\beta|} \partial_{\beta}\{\mathbf{I}-\mathbf{P}\} f\right|_{w} d x \\
& \leq \sum_{\left|\beta_{1}\right| \leq|\beta|} \int_{\mathbf{R}^{3}}\left(|a|^{2}+|b|^{2}+|c|^{2}\right)\left|w^{|\beta|} \partial_{\beta}\{\mathbf{I}-\mathbf{P}\} f\right|_{w} d x \\
& \leq C\left\|a^{2}+|b|^{2}+c^{2}\right\|\left\|\mid w^{|\beta|} \partial_{\beta}\{\mathbf{I}-\mathbf{P}\} f\right\|_{w} .
\end{aligned}
$$


The similar argument as used in (4.4) implies that the first factor of the above is bounded by $C\left|\left\|f\left|\left\|_{|\beta|}|||f|\right\|_{w,|\beta|}\right.\right.\right.$. Thus this term is bounded by $\left.\left.\left.C\right|\right\| f\right|||_{|\beta|}|||f| \|_{w,|\beta|}^{2}$.

For the last term of (5.8), we directly apply Lemma 2.5 with $\theta=|\beta|$ for the Boltzmann equation to get

$$
\begin{aligned}
& \left(w^{2|\beta|} \partial_{\beta} \Gamma(\{\mathbf{I}-\mathbf{P}\} f,\{\mathbf{I}-\mathbf{P}\} f), \partial_{\beta}\{\mathbf{I}-\mathbf{P}\} f\right) \\
& \leq C\left[\sum_{\left|\alpha_{i}\right|+\left|\beta_{i}\right| \leq N}\left\|w^{\left|\beta_{i}\right|} \partial_{\beta_{i}}^{\alpha_{i}}\{\mathbf{I}-\mathbf{P}\} f\right\|\right]\left\|w^{\left|\beta_{2}\right|} \partial_{\beta_{2}}\{\mathbf{I}-\mathbf{P}\} f\right\|_{\nu}\left\|w^{|\beta|} \partial_{\beta}\{\mathbf{I}-\mathbf{P}\} f\right\|_{\nu} \\
& \leq C\left|\left\|f|\|||\beta||| f||_{\nu,|\beta|}^{2} .\right.\right.
\end{aligned}
$$

For the last term of (5.8) in the Landau equation, we apply (3.38) in Lemma 3.4 with $\theta=|\beta|$ and the Sobolev embedding theorem to get

$$
\begin{aligned}
& \left(w^{2|\beta|} \partial_{\beta} \Gamma(\{\mathbf{I}-\mathbf{P}\} f,\{\mathbf{I}-\mathbf{P}\} f), \partial_{\beta}\{\mathbf{I}-\mathbf{P}\} f\right) \\
& \leq C\left[\left\{\sum\left\|\partial_{\beta_{1}}^{\alpha_{1}}\{\mathbf{I}-\mathbf{P}\} f\right\|_{\left|\beta_{1}\right|}\right\}\left\{\sum\left\|\partial_{\beta_{2}}^{\alpha_{2}}\{\mathbf{I}-\mathbf{P}\} f\right\|_{\sigma,\left|\beta_{2}\right|}\right\}\right. \\
& \left.\quad+\left\{\sum\left\|\partial_{\beta_{1}}^{\alpha_{1}}\{\mathbf{I}-\mathbf{P}\} f\right\|_{\left|\beta_{1}\right|}\right\}\left\{\sum\left\|\partial_{\beta_{2}}^{\alpha_{2}}\{\mathbf{I}-\mathbf{P}\} f\right\|_{\sigma,\left|\beta_{2}\right|}\right\}\right]\left\|\partial_{\beta}\{\mathbf{I}-\mathbf{P}\} f\right\|_{\sigma,|\beta|} \\
& \leq C\left|\left\|f \left|\left\|_{|\beta|} \mid\right\| f \|_{\sigma,|\beta|}^{2},\right.\right.\right.
\end{aligned}
$$

where the summations are over $\left|\alpha_{1}\right|+\left|\beta_{1}\right| \leq N$ and $\left|\alpha_{2}\right|+\left|\beta_{2}\right| \leq N$ respectively.

If we collect the above inequalities and choose $\eta>0$ small enough, we have

$$
\begin{aligned}
& \frac{1}{2} \frac{d}{d t} \sum_{|\beta| \neq 0}\left\|\partial_{\beta}\{\mathbf{I}-\mathbf{P}\} f\right\|_{|\beta|}^{2}+\sum_{|\beta| \neq 0}\left\|\partial_{\beta}\{\mathbf{I}-\mathbf{P}\} f\right\|_{w,|\beta|}^{2} \\
& \leq C\left|\left\|f\left|\|\|_{|\beta|}\right|\right\| f\right|\left\|_{w,|\beta|}^{2}+C \sum_{\left|\alpha_{1}\right|=1}\right\| \partial^{\alpha_{1}} f\left\|_{w}^{2}+C\right\|\{\mathbf{I}-\mathbf{P}\} f \|_{w}^{2} .
\end{aligned}
$$

The standard energy estimate for $\beta=0$ is

$$
\frac{1}{2} \frac{d}{d t}\|f\|^{2}+(L f, f)=(\Gamma(f, f), f)
$$

It is easily known that

$$
\langle\Gamma(f, f), \mathbf{P} f\rangle=0 .
$$

A similar argument to (5.2) implies that

$$
(\Gamma(f, f),\{\mathbf{I}-\mathbf{P}\} f) \leq C|||f|\left\|_{|\beta|}|||f|\right\|_{w,|\beta|}^{2} .
$$

Therefore, we get

$$
\frac{1}{2} \frac{d}{d t}\|f\|^{2}+\delta_{1}\|\{\mathbf{I}-\mathbf{P}\} f\|_{w}^{2} \leq C\left|\left\|f\left|\|\|_{|\beta|}\right|\right\| f\right| \|_{w,|\beta|}^{2} .
$$

In the following we consider $\partial_{\beta}^{\alpha}$ derivatives of (1.3) where $|\alpha| \neq 0$, and $|\beta| \neq 0$. Assuming $|\alpha|+|\beta| \leq N$ and taking $\partial_{\beta}^{\alpha}$ derivatives of (1.3), we obtain

$$
\left[\partial_{t}+v \cdot \nabla_{x}\right] \partial_{\beta}^{\alpha} f+\sum_{\left|\beta_{1}\right|=1} C_{\beta}^{\beta_{1}} \partial_{\beta_{1}} v \cdot \nabla_{x} \partial_{\beta-\beta_{1}}^{\alpha} f+\partial_{\beta}\left[L \partial^{\alpha} f\right]=\partial_{\beta}^{\alpha} \Gamma(f, f) .
$$

We take the inner product of (5.11) with $w^{2|\beta|} \partial_{\beta}^{\alpha} f$ over $\mathbf{R}^{3} \times \mathbf{R}^{3}$ and estimate this inner product term by term. 
Now we consider the nonlinear collision term $\partial_{\beta}^{\alpha} \Gamma(f, f)$. We first decompose the inner product of $\partial_{\beta}^{\alpha} f$ with the collision term, $\left(w^{2|\beta|} \partial_{\beta}^{\alpha} \Gamma(f, f), \partial_{\beta}^{\alpha} f\right)$, as follows:

$$
\begin{array}{r}
\left(w^{2|\beta|} \partial_{\beta}^{\alpha} \Gamma(\mathbf{P} f,\{\mathbf{I}-\mathbf{P}\} f), \partial_{\beta}^{\alpha} f\right)+\left(w^{2|\beta|} \partial_{\beta}^{\alpha} \Gamma(\{\mathbf{I}-\mathbf{P}\} f, \mathbf{P} f), \partial_{\beta}^{\alpha} f\right) \\
+\left(w^{2|\beta|} \partial_{\beta}^{\alpha} \Gamma(\mathbf{P} f, \mathbf{P} f), \partial_{\beta}^{\alpha} f\right)+\left(w^{2|\beta|} \partial_{\beta}^{\alpha} \Gamma(\{\mathbf{I}-\mathbf{P}\} f,\{\mathbf{I}-\mathbf{P}\} f), \partial_{\beta}^{\alpha} f\right) .
\end{array}
$$

For the first term of (5.12), we directly apply Corollary 2.1 and Corollary 3.1 to get

$$
\begin{aligned}
& \left(w^{2|\beta|} \partial_{\beta}^{\alpha} \Gamma(\mathbf{P} f,\{\mathbf{I}-\mathbf{P}\} f), \partial_{\beta}^{\alpha} f\right) \\
\leq & C \sum \int_{\mathbf{R}^{3}}\left(\left|\partial^{\alpha_{1}} a\right|+\left|\partial^{\alpha_{1}} b\right|+\left|\partial^{\alpha_{1}} c\right|\right)\left|w^{|\beta|} \partial_{\beta_{2}}^{\alpha_{2}}\{\mathbf{I}-\mathbf{P}\} f\right|_{w}\left|w^{|\beta|} \partial_{\beta}^{\alpha} f\right|_{w} d x \\
\leq & C \sum \sup _{x}\left(\left|\partial^{\alpha_{1}} a\right|+\left|\partial^{\alpha_{1}} b\right|+\left|\partial^{\alpha_{1}} c\right|\right) \int_{\mathbf{R}^{3}}\left|w^{|\beta|} \partial_{\beta_{2}}^{\alpha_{2}}\{\mathbf{I}-\mathbf{P}\} f\right|_{w}\left|w^{|\beta|} \partial_{\beta}^{\alpha} f\right|_{w} d x \\
\leq & C\left|\left\|f\left|\left\|\left.\right|_{|\beta|}\right\| w^{\left|\beta_{2}\right|} \partial_{\beta_{2}}^{\alpha_{2}}\{\mathbf{I}-\mathbf{P}\} f\left\|_{w}\right\| w^{|\beta|} \partial_{\beta}^{\alpha} f\left\|_{w} \leq C|||f|\right\|_{|\beta|} \cdot\right||| f \mid\right\|_{w,|\beta|}^{2},\right.
\end{aligned}
$$

where without loss of generality we have assumed that $\left|\alpha_{2}\right|+\left|\beta_{2}\right| \geq N / 2$. For the second and third terms of (5.12), they have the same upper bound as the above by similar arguments.

For the last term of (5.12), we directly apply Lemma 2.3 and (3.38) in Lemma 3.4 with $\theta=|\beta|$ to get

$$
\begin{aligned}
& \left(w^{2|\beta|} \partial_{\beta}^{\alpha} \Gamma(\{\mathbf{I}-\mathbf{P}\} f,\{\mathbf{I}-\mathbf{P}\} f), \partial_{\beta}^{\alpha} f\right) \\
\leq & C\left|\left\|f\left|\|\|_{|\beta|}\left\|w^{\left|\beta_{2}\right|} \partial_{\beta_{2}}^{\alpha_{2}}\{\mathbf{I}-\mathbf{P}\} f\right\|_{w}\left\|w^{|\beta|} \partial_{\beta}^{\alpha} f\right\|_{w} \leq C\right|\right\| f\right|\left\|_{|\beta|} \cdot \mid\right\| f \|_{w,|\beta|}^{2} .
\end{aligned}
$$

Using a similar method as above, the inner product of the second term on the left-hand side of (5.11) is bounded by

$$
\sum_{\left|\beta_{1}\right|=1}\left(w^{2|\beta|} \partial_{\beta_{1}} v_{j} \cdot \partial^{j} \partial_{\beta-\beta_{1}}^{\alpha} f, \partial_{\beta}^{\alpha} f\right) \leq C_{\eta} \sum_{\left|\beta_{1}\right|=1}\left\|w^{\left|\beta_{1}\right|} \partial^{j} \partial_{\beta_{1}}^{\alpha} f\right\|_{w}^{2}+\eta\left\|w^{|\beta|} \partial_{\beta}^{\alpha} f\right\|_{w}^{2} .
$$

From Lemma 2.4 and Lemma 3.4, we deduce that, for $\eta>0$ small enough, the inner product of the third term on the left-hand side of (5.11) is controlled by

$$
\left(w^{2|\beta|} \partial_{\beta} L \partial^{\alpha} f, \partial_{\beta}^{\alpha} f\right) \geq\left\|w^{|\beta|} \partial_{\beta}^{\alpha} f\right\|_{w}^{2}-\eta \sum_{\beta_{1} \leq \beta}\left\|w^{\left|\beta_{1}\right|} \partial_{\beta_{1}}^{\alpha} f\right\|_{w}^{2}-C_{\eta}\left\|\partial^{\alpha} f\right\|_{w}^{2} .
$$

Thus, for any $0<|\beta| \leq N$, we have

$$
\begin{aligned}
& \frac{1}{2} \frac{d}{d t}\left\|w^{|\beta|} \partial_{\beta}^{\alpha} f\right\|^{2}+\delta_{N}\left\|w^{|\beta|} \partial_{\beta}^{\alpha} f\right\|_{w}^{2} \leq C\left|\left\|f\left|\left\|_{|\beta|} \cdot\right\|\right||f|\right\|_{w,|\beta|}^{2}\right. \\
& \quad+C \sum_{|\alpha| \neq 0}\left\|\partial^{\alpha} f\right\|_{w}^{2}+C_{\eta} \sum_{\left|\beta_{1}\right|=1}\left\|w^{\left|\beta_{1}\right|} \partial^{j} \partial_{\beta_{1}}^{\alpha} f\right\|_{w}^{2}+\eta \sum_{\beta_{1}<\beta}\left\|w^{\left|\beta_{1}\right|} \partial_{\beta_{1}}^{\alpha} f\right\|_{w}^{2} .
\end{aligned}
$$


Thus, for any $0<|\beta| \leq N$ and suitable linear combination of (5.13) depending on $|\beta|$, we have that

$$
\begin{aligned}
& \sum_{\alpha, \beta \in \Lambda}\left[\frac{1}{2} \frac{d}{d t}\left\|\partial_{\beta}^{\alpha} f\right\|_{|\beta|}^{2}+\delta_{N}\left\|\partial_{\beta}^{\alpha} f\right\|_{w,|\beta|}^{2}\right] \\
& \leq C\left|\left\|f \left|\left\|_ { | \beta | } \cdot \left|\|f \mid\|_{w,|\beta|}^{2}+C \sum_{|\alpha| \neq 0}\left\|\partial^{\alpha} f\right\|_{w}^{2},\right.\right.\right.\right.\right.
\end{aligned}
$$

where the indices set $\Lambda=\{|\alpha|+|\beta| \leq N,|\alpha| \neq 0,|\beta| \neq 0\}$.

A suitable linear combination of (5.3), (5.9), (5.10) and (5.14) yields the following estimates:

$$
\begin{gathered}
\frac{1}{2} \frac{d}{d t}\left[C_{1} \sum_{\alpha, \beta \in \Lambda}\left\|\partial_{\beta}^{\alpha} f\right\|_{|\beta|}^{2}+C_{2} \sum_{0<|\beta| \leq N}\left\|\partial_{\beta}\{\mathbf{I}-\mathbf{P}\} f\right\|_{|\beta|}^{2}\right. \\
\left.+C_{3} \sum_{0<|\alpha| \leq N}\left\|\partial^{\alpha} f\right\|^{2}+C_{4}\|f\|^{2}-C_{5} \int_{\mathbf{R}^{3}} \nabla \cdot b c d x\right] \\
+\delta\left[\sum_{|\beta| \leq N}\left\|\partial_{\beta}\{\mathbf{I}-\mathbf{P}\} f\right\|_{w,|\beta|}^{2}+\sum_{|\alpha|+|\beta| \leq N,|\alpha| \neq 0}\left\|\partial_{\beta}^{\alpha} f(t)\right\|_{w,|\beta|}^{2}\right] \\
\leq C\left|\left\|f \left|\left\|_ { | \beta | } \cdot \left|\|f \mid\|_{w,|\beta|}^{2} .\right.\right.\right.\right.\right.
\end{gathered}
$$

Here $C_{1}, C_{2}, C_{3}, C_{4}, C_{5}$ and $\delta$ are positive constants where $C_{4}$ is sufficiently large.

We can choose $C_{4}$ large enough such that, for some constant $C_{6}>0$, we have

$$
\begin{aligned}
y(t) \equiv C_{1} & \sum_{\alpha, \beta \in \Lambda}\left\|\partial_{\beta}^{\alpha} f\right\|_{|\beta|}^{2}+C_{2} \sum_{0<|\beta| \leq N}\left\|\partial_{\beta}\{\mathbf{I}-\mathbf{P}\} f\right\|_{|\beta|}^{2} \\
& +C_{3} \sum_{0<|\alpha| \leq N}\left\|\partial^{\alpha} f\right\|^{2}+C_{4}\|f\|^{2}-C_{5} \int_{\mathbf{R}^{3}} \nabla \cdot b c d x \geq C_{6}\left|\|f \mid\|_{|\beta|}^{2} .\right.
\end{aligned}
$$

We easily obtain that $y(t) \leq C_{7}\left|\|f \mid\|_{|\beta|}^{2}\right.$ for some constant $C_{7}>0$. Therefore, (5.15) is rewritten as

$$
\frac{1}{2} y^{\prime}(t)+\delta|||f|\left\|_{w,|\beta|}^{2} \leq C|||f|\right\|_{|\beta|} \cdot|||f| \|_{w,|\beta|}^{2} \cdot
$$

To proceed, we define

$$
M \equiv \min \left\{\frac{\delta}{2 C}, M_{0}\right\}>0,
$$

and choose initial data so that $y(0) \leq \frac{M}{2}<M_{0}$. From Lemma 5.1 we may choose $T>0$ so that

$$
T=\sup \{t: y(t) \leq M\}>0
$$

since $y(t)$ is continuous. Notice that for $0 \leq t \leq T, M \leq M_{0}$. Thus, the small amplitude assumption (4.6) is valid. We apply (5.16) to get, for $0 \leq t \leq T$, that

$$
\frac{1}{2} y^{\prime}(t)+\delta \||| f||_{w,|\beta|}^{2} \leq\left. C|||f||| \cdot|||f|\right|_{w,|\beta|} ^{2} \leq \frac{\delta}{2}|||f|||_{w,|\beta|}^{2} .
$$

Therefore, an integration over $0 \leq t \leq T$ yields

$$
y(t)+\delta \int_{0}^{t}\left\||f(s) \||_{w,|\beta|}^{2} d s \leq y(0) \leq \frac{M}{2}<M .\right.
$$


Since $y(t)$ is continuous in $t$, this implies that $y(T) \leq M$ if $T<\infty$. This is a contradiction to the definition of $T$. Hence $T=\infty$. It is straightforward to verify that, for any $s>0$, $\mathcal{E}(f(s)) \leq C_{0} \mathcal{E}\left(f_{0}\right)$ by $(5.17)$.

Acknowledgements. H. Yu thanks Professor Dafermos, Professor Tong Yang and Professor Yan Guo for their many valuable suggestions. H. Yu also thanks Robert Strain for sending their papers [23, 24, 25, to him. The research of L. Hsiao was supported by the Funds of NSF of China (Grant No. 10431060). The research of H. Yu was supported by the Young Scientists Funds of NSF of China (Grant No. 10601018) and the Scientific Funds of Guangdong Province (Grant No. 06300210).

\section{REFERENCES}

[1] Alexandre, R. and Villani, C., On the Boltzmann equation for long-range interactions. Comm. Pure Appl. Math. 55 (1) (2002), 30-70. MR1857879 (2002f:82026)

[2] Alexandre, R. and Villani, C., On the Landau approximation in plasma physics. Ann. Inst. H. Poincaré Anal. Non Linéaire 21 (1) (2004), 61-95. MR2037247(2005f:82126)

[3] Caflisch, R., The Boltzmann equation with a soft potential, I. Linear, spatially homogeneous. Comm. Math. Phys. 74 (1980), 71-95. MR.575897 (82j:82040a)

[4] Caflisch, R., The Boltzmann equation with a soft potential, II. Nonlinear, spatially periodic. Comm. Math. Phys. 74 (1980), 97-107. MR576265 (82j:82040b)

[5] Cercignani, C., The Boltzmann equation and its applications. Springer-Verlag, New York, 1988. MR.1313028 (95i:82082)

[6] Degond, P. and Lemou, M., Dispersion relations for the linearized Fokker-Planck equation. Arch. Rat. Mech. Anal. 138 (2)(1997), 137-167. MR1463805 (99f:82051)

[7] Desvillettes, L. and Villani, C., On the spatially homogeneous Landau equation for hard potentials (I-II). Comm. P.D.E. 25 (1-2), (2000), 179-298. MR1737547 (2001c:82065)

[8] Diperna, R. J. and Lions, P. L., On the Cauchy problem for Boltzmann equations: Global existence and weak stability, Ann. Math. 130 (1989), 321-366. MR1014927(90k:82045)

[9] Glassey, R. T., The Cauchy problem in kinetic theory. Society for Industrial and Applied Mathematics (SIAM), Philadelphia, PA, 1996. MR.1379589 (97i:82070)

[10] Grad, H., Asymptotic theory of the Boltzmann equation II, in Rarefied Gas Dynamics, J. A. Laurmann, ed., Academic Press, New York, 1963, 26-59. MR0156656 (27:6577)

[11] Guo, Y., The Vlasov-Poisson-Boltzmann system near vacuum. Comm. Math. Phys. 218 (2) (2001), 293-313. MR1828983 (2002e:35055)

[12] Guo, Y., The Landau Equation in periodic box. Comm. Math. Phys. 231 (2002), 391-434. MR $1946444(2004 \mathrm{c}: 82121)$

[13] Guo, Y., The Vlasov-Poisson-Boltzmann system near Maxwellians. Comm. Pure Appl. Math. 55 (9) (2002), 1104-1135. MR1908664 (2003b:82050)

[14] Guo, Y., The Vlasov-Maxwell-Boltzmann system near Maxwellians. Invent. Math. 153 (3) (2003), 593-630. MR2000470 (2004m:82123)

[15] Guo, Y., Classical solutions to the Boltzmann equation for molecules with angular cutoff. Arch. Rat. Mech. Anal. 169 (2003), 305-353. MR2013332 (2004i:82054)

[16] Guo, Y., The Boltzmann equation in the whole space. Indiana Univ. Math. J. 53 (4) (2004), 10811094. MR2095473 (2005g:35028)

[17] Hsiao, L. and Yu, H.-J., Global classical solution to the initial value problem for the relativistic Landau equation. J. Diff. Eqn. 228 (2006), 641-660.

[18] Lemou, M., Linearized quantum and relativistic Fokker-Planck-Landau equations. Math. Methods Appl. Sci. 23 (12) (2000), 1093-1119. MR1773932(2001g:82079)

[19] Lions, P. L., On the kinetic equations. Proceedings of ICM I-II (Kyoto, 1990), 1173-1185. MR 1159302 (93c:82051)

[20] Lions, P. L., On Boltzmann and Landau equations. Phil. Trans. R. Soc. Lon. A 346 (1994), 191-204. MR $1278244(95 \mathrm{~d}: 82050)$ 
[21] Liu, T.-P., Yang, T., and Yu, S.-H., Energy method for the Boltzmann equation. Physica D 188 (3-4) (2004), 178-192. MR2043729 (2005a:82091)

[22] Liu, T.-P. and Yu, S.-H., Boltzmann equation: Micro-macro decompositions and positivity of shock profiles. Commun. Math. Phys. 246 (1) (2004), 133-179. MR2044894 (2005f:82101)

[23] Strain, R. M. and Guo, Y., Stability of the relativistic Maxwellian in a collisional plasma. Comm. Math. Phys. 251 (2) (2004), 263-320. MR2100057 (2005m:82155)

[24] Strain, R. M. and Guo Y., Almost exponential decay near Maxwellian. Comm. P.D.E. 31 (2006), 417-429. MR2209761 (2006m:82042)

[25] Strain, R. M. and Guo, Y., Exponential decay for soft potentials near Maxwellian. preprint, 2005.

[26] Truesdell, C. and Muncaster, R. G., Fundamentals Maxwell's Kinetic Theory of a simple monoatomic gas. Academic Press, New York, 1980. MR554086 (81f:80001)

[27] Ukai, S., On the existence of global solutions of mixed problem for non-linear Boltzmann equation. Proc. Japan Acad. 50 (1974), 179-184. MR0363332(50:15770)

[28] Ukai, S. and Asano, K., On the Cauchy problem of Boltzmann equation with a soft potential, Publ. Res. Inst. Math. Sci. 18 (1982), 477-519. MR677262 (84h:82048)

[29] Villani, C., On a new class of weak solutions to the spatial homogeneous Boltzmann and Landau equations. Arch. Rat. Mech. Anal. 143 (3) (1998), 237-307. MR1650006 (99j:82065)

[30] Villani, C., On the Landau equation: Weak stability, global existence. Adv. Diff. Eq. 1 (5) (1996), 793-816. MR 1392006 (97e:82048)

[31] Villani, C., A survey of mathematical topics in kinetic theory. To appear in Handbook of fluid mechanics, S. Friedlander and D. Serre, Eds.

[32] Yang, T., Yu, H.-J., and Zhao, H.-J., Cauchy problem for the Vlasov-Poisson-Boltzmann system, Arch. Rat. Mech. Anal. 182 (2006), 415-470. MR2276498

[33] Yu, H.-J., Global solution of the Vlasov-Poisson-Landau systems near Maxwellians with small amplitude, J. Part. Diff. Eqn. 17 (2) (2004), 173-192. MR2060788 (2005f:35042)

[34] Yu, H.-J., Global classical solution of the Vlasov-Maxwell-Landau system near Maxwellians, $J$. Math. Phys. 11 (45) (2004), 4360-4376. MR2098143(2005h:82109)

[35] Yu, H.-J., Existence and exponential decay of global solution to the Boltzmann equation near Maxwellians, Math. Models Methods Appl. Sci. 15 (3)(2005), 483-505. MR2126140 (2005m:35038)

[36] Zhan, M., Local existence of classical solutions to the Landau equations. Transport Theory Statist. Phys. 23 (4) (1994), 479-499. MR1264848 (95c:35203)

[37] Zhan, M., Local existence of solutions to the Landau-Maxwell system. Math. Methods Appl. Sci. 17 (8) (1994), 613-641. MR1280648 (95h:35228) 